\title{
Measuring the running top-quark mass
}

\author{
U. Langenfeld, ${ }^{1}$ S. Moch, ${ }^{1}$ and P. Uwer ${ }^{2}$ \\ ${ }^{1}$ Deutsches Elektronensynchrotron DESY, Platanenallee 6, D15738 Zeuthen, Germany \\ ${ }^{2}$ Institut für Physik, Humboldt-Universität zu Berlin, Newtonstraße 15, D12489 Berlin, Germany
}

(Received 9 July 2009; published 9 September 2009)

\begin{abstract}
We present the first direct determination of the running top-quark mass based on the total cross section of top-quark pair production as measured at the Tevatron. Our theory prediction for the cross section includes various next-to-next-to-leading order QCD contributions, in particular, all logarithmically enhanced terms near threshold, the Coulomb corrections at two loops and all explicitly scale-dependent terms at next-to-next-to-leading order accuracy. The result allows for an exact and independent variation of the renormalization and factorization scales. For Tevatron and LHC we study its dependence on all scales, on the parton luminosity and on the top-quark mass using both the conventional pole mass definition as well as the running mass in the $\overline{\mathrm{MS}}$ scheme. We extract for the top quark an $\overline{\mathrm{MS}}$ mass of $m(\mu=m)=160.0_{-3.2}^{+3.3} \mathrm{GeV}$, which corresponds to a pole mass of $m_{t}=168.9_{-3.4}^{+3.5} \mathrm{GeV}$.

DOI: 10.1103/PhysRevD.80.054009

PACS numbers: $12.38 . \mathrm{Bx}, 13.85 . \mathrm{Ni}$
\end{abstract}

\section{INTRODUCTION}

The top quark is the heaviest elementary particle discovered so far and it is likely to be the most sensitive probe of the electroweak symmetry breaking. This is reflected in the fact that in many extensions of the standard model the top quark plays a special role. The precise measurement of top-quark properties is thus an important task for the Large Hadron Collider (LHC) (see e.g. Refs. [1,2]). One of the most basic quantities in that respect is the total cross section which is currently measured at the Tevatron and will be measured at the LHC. The precise measurements aimed for at the LHC are asking for an equally precise theoretical prediction to compare with. In this paper, we update and extend the predictions of Refs. [3,4] for the total hadronic cross section of top-quark pairs and its associated theoretical uncertainty. Related recent studies have also appeared in Refs. [5-7]. As a novel aspect of this paper, we employ the $\overline{\mathrm{MS}}$ definition for the top-quark mass and present the total cross section as a function of the running mass. This allows the direct determination of an $\overline{\mathrm{MS}}$ mass from Tevatron measurements for the total cross section [8].

We start by recalling the relevant formulas for the total cross section of top-quark hadroproduction within perturbative quantum chromodynamics (QCD):

$$
\sigma_{p p \rightarrow \bar{t} X}\left(S, m_{t}^{2}\right)=\frac{\alpha_{s}^{2}}{m_{t}^{2}} \sum_{i, j=q, \bar{q}, g} \int_{4 m_{t}^{2}}^{S} d s L_{i j}\left(s, S, \mu_{f}^{2}\right) f_{i j}(\rho, M, R),
$$

$$
L_{i j}\left(s, S, \mu_{f}^{2}\right)=\frac{1}{S} \int_{s}^{S} \frac{d \hat{s}}{\hat{s}} \phi_{i / p}\left(\frac{\hat{s}}{S}, \mu_{f}^{2}\right) \phi_{j / p}\left(\frac{s}{\hat{s}}, \mu_{f}^{2}\right),
$$

where $S$ and $m_{t}$ denote the hadronic center-of-mass energy squared and the top-quark mass (here taken to be the pole mass), respectively, while $L_{i j}$ is the usual definition of the parton luminosity with the parton distributions (PDFs) $\phi_{i / p}$ at the factorization scale $\mu_{f}$. The scaling functions $f_{i j}$ parametrize the hard partonic scattering process. They depend only on dimensionless ratios of $m_{t}, \mu_{f}$, the renormalization scale $\mu_{r}$ and the partonic center-of-mass energy squared $s$, with the definitions $\rho=4 m_{t}^{2} / s, R=\mu_{r}^{2} / \mu_{f}^{2}$ and $M=\mu_{f}^{2} / m_{t}^{2}$. The radiative corrections to the scaling functions $f_{i j}$ at the next-to-leading order (NLO) [9-11] are long known and recently even the complete analytic expressions have become available [12]. In order to quantify the theory uncertainty also the next-to-next-to-leading order (NNLO) must be included. Presently, these NNLO corrections $[3,4]$ are approximated by the complete tower of the Sudakov logarithms and including all two-loop Coulomb corrections.

In the present study we include consistently the channels, $q \bar{q}, g g$ and $g q$ through NNLO and we provide parametrizations for all necessary scaling functions in the standard $\overline{\mathrm{MS}}$ scheme for mass factorization. This allows for an easy handling in phenomenological applications. Our phenomenological study reflects the latest measured value for the top-quark mass [13], $m_{t}=173.1_{-1.3}^{+1.3} \mathrm{GeV}$, and employs new PDF sets $[7,14]$. Let us briefly summarize the key aspects of our update with respect to Refs. [3,4]:

(i) We use exact dependence on the renormalization and factorization scale. This allows for an independent variation of $\mu_{r}$ and $\mu_{f}$ (extending Ref. [15]) and is commonly considered as more reliable to establish the theoretical uncertainty of perturbative predictions (see e.g. Ref. [16]).

(ii) We perform the singlet-octet color decomposition consistently when matching our threshold expansion at NLO using results of Refs. [17-19]. The numerical impact on phenomenology at LHC and Tevatron turns out to be negligible, though. 
(iii) We discuss those residual systematical uncertainties of our predictions for LHC and Tevatron, which are inherent in the approach based on threshold resummation and we comment on the size of unknown corrections.

(iv) We quantify the numerical impact of other known effects on the total cross section, such as the electroweak radiative corrections at NLO [20-22] and bound state corrections in QCD at threshold $[18,19]$.

(v) We study the dependence of the total cross section on the definition of the mass parameter. For the conversion of the conventionally used pole mass $m_{t}$ [see Eq. (1)] and the scale-dependent $\overline{\mathrm{MS}}$ mass $m\left(\mu_{r}\right)$ we exploit well-known relations to NNLO [23] (see also Refs. $[24,25])$. We investigate the apparent convergence of both definitions, $m_{t}$ and $m\left(\mu_{r}\right)$, in perturbation theory through NNLO.

We also employ the analytic results for the NLO scaling functions [12]. As a net effect these lead to small improvements in the $g q$ - and the $g g$-channel contributions of our NNLO prediction.

\section{THEORETICAL SETUP}

The perturbative expansion of the scaling functions $f_{i j}$ in the strong coupling $\alpha_{s}$ up to two loops around $M=R=$ 1, i.e. $m_{t}=\mu_{r}=\mu_{f}$, reads

$$
f_{i j}(\rho, 1,1)=f_{i j}^{(0)}(\rho)+4 \pi \alpha_{s} f_{i j}^{(10)}(\rho)+\left(4 \pi \alpha_{s}\right)^{2} f_{i j}^{(20)}(\rho),
$$

and the functions $f_{i j}^{(0)}, f_{i j}^{(10)}$ and $f_{i j}^{(20)}$ contain, at each order in $\alpha_{s}$, genuinely new information to be calculated from first principles in perturbation theory. At the Born level,

$$
\begin{gathered}
f_{q \bar{q}}^{(0)}=\frac{\pi \beta \rho}{27}[2+\rho], \\
f_{g q}^{(0)}=0, \\
f_{g g}^{(0)}=\frac{\pi \beta \rho}{192}\left[\frac{1}{\beta}\left(\rho^{2}+16 \rho+16\right) \ln \left(\frac{1+\beta}{1-\beta}\right)-28-31 \rho\right],
\end{gathered}
$$

where $\beta$ is the heavy quark velocity with $\beta=\sqrt{1-\rho}$. At NLO the known functions $f_{i j}^{(10)}$ can be described through parametrizations which are accurate at the per mille level. Our one-loop fits use the following ansatz:

$$
\begin{aligned}
f_{q \bar{q}}^{(10)}= & \frac{\rho \beta}{36 \pi}\left[\frac{32}{3} \ln ^{2} \beta+\left(32 \ln 2-\frac{82}{3}\right) \ln \beta-\frac{1}{12} \frac{\pi^{2}}{\beta}\right] \\
& +\beta \rho a_{0}^{q q}+h\left(\beta, a_{1}, \ldots, a_{17}\right) \\
& +\frac{1}{8 \pi^{2}}\left(n_{f}-4\right) f_{q \bar{q}}^{(0)}\left[\frac{4}{3} \ln 2-\frac{2}{3} \ln \rho-\frac{10}{9}\right],
\end{aligned}
$$

$$
\begin{aligned}
f_{g q}^{(10)}= & \frac{1}{16 \pi} \beta^{3}\left[\frac{5}{9} \ln \beta+\frac{5}{6} \ln 2-\frac{73}{108}\right] \\
& +h_{g q}^{(a)}\left(\beta, a_{1}, \ldots, a_{15}\right),
\end{aligned}
$$

$$
\begin{aligned}
f_{g g}^{(10)}= & \frac{7 \beta}{768 \pi}\left[24 \ln ^{2} \beta+\left(72 \ln 2-\frac{366}{7}\right) \ln \beta+\frac{11}{84} \frac{\pi^{2}}{\beta}\right] \\
& +\beta a_{0}^{g g}+h\left(\beta, a_{1}, \ldots, a_{17}\right)+\left(n_{f}-4\right) \frac{\rho^{2}}{1024 \pi} \\
& \times\left[\ln \left(\frac{1+\beta}{1-\beta}\right)-2 \beta\right],
\end{aligned}
$$

where $n_{f}$ denotes the number of light quarks and we have kept the complete dependence on $n_{f}$ in all parametrizations manifest. The Sudakov logarithms $\ln \beta$ at threshold and the Coulomb corrections $(\sim 1 / \beta)$ in Eqs. (7)-(9) are exact [9]. The constants $a_{0}^{i j}$ read

$$
\begin{aligned}
a_{0}^{q q} & =\frac{299}{324 \pi}-\frac{43}{1296} \pi-\frac{121}{108} \frac{\ln 2}{\pi}+\frac{16}{27} \frac{\ln ^{2} 2}{\pi} \\
& \approx 0.03294734,
\end{aligned}
$$

$$
\begin{aligned}
a_{0}^{g g} & =\frac{1111}{2304 \pi}-\frac{283}{18432} \pi-\frac{89}{128} \frac{\ln 2}{\pi}+\frac{7}{16} \frac{\ln ^{2} 2}{\pi} \\
& \approx 0.01875287 .
\end{aligned}
$$

They are known from the computation of the NLO QCD corrections to hadroproduction of quarkonium [17] (see also Refs. [18,19]), where also details of the decomposition of $a_{0}^{g g}$ for color-singlet and color-octet states can be found. The constants $a_{0}^{i j}$ in Eqs. (10) and (11) emerge from Refs. [17-19] by means of a simple Mellin transformation and agree with the values quoted in Ref. [12]. The coefficients of the functions $h\left(\beta, a_{1}, \ldots, a_{17}\right)$ and $h_{g q}^{(a)}\left(\beta, a_{1}, \ldots, a_{15}\right)$ in Eqs. (7)-(9) are determined in a fit to the analytic expressions of Ref. [12]. Near threshold we have $h(\beta)=\mathcal{O}\left(\beta^{2}\right)$. More details are given in Eqs. (A7) and (A8). Because of the larger number of parameters in the fit functions, it is evident that Eqs. (7)-(9) supersede earlier parametrizations [9] with respect to accuracy.

At the two-loop level we know the complete tower of Sudakov logarithms, $\ln ^{k} \beta$ with $k=1, \ldots, 4$, for the functions $f_{q \bar{q}}^{(20)}$ and $f_{g g}^{(20)}$ and, in addition, also the complete Coulomb contributions, $\sim 1 / \beta^{2}, 1 / \beta$. The channel $g q$ is power suppressed near threshold relative to $q \bar{q}$ and $g g$. However, extending soft gluon resummation to power suppressed quantities (see e.g. Refs. [26,27]) and using Eq. (8), we can determine (at least) the leading term $\sim \ln ^{3} \beta$ of the function $f_{g q}^{(20)}$. We find 


$$
\begin{aligned}
& f_{q \bar{q}}^{(20)}= \frac{f_{q \bar{q}}^{(0)}}{\left(16 \pi^{2}\right)^{2}}\left[\frac{8192}{9} \ln ^{4} \beta+\left(-\frac{15872}{3}+\frac{16384}{3} \ln 2+\frac{1024}{27} n_{f}\right) \ln ^{3} \beta\right. \\
&+\left(1046.4831-90.838135 n_{f}-140.36771 \frac{1}{\beta}\right) \ln ^{2} \beta+\left(1029.8687-2.8903919 n_{f}-2 D_{Q \bar{Q}}^{(2)}\right. \\
&\left.\left.+\left(54.038454-4.3864908 n_{f}\right) \frac{1}{\beta}\right) \ln \beta+3.6077441 \frac{1}{\beta^{2}}+\left(7.3996963+0.61492528 n_{f}\right) \frac{1}{\beta}+C_{q \bar{q}}^{(2)}\right] \\
& f_{g q}^{(20)}=\frac{\beta^{3}}{\left(16 \pi^{2}\right)^{2}} \frac{65 \pi}{54} \ln ^{3}\left(8 \beta^{2}\right), \\
& f_{g g}^{(20)}=\frac{f_{g g}^{(0)}}{\left(16 \pi^{2}\right)^{2}}\left[4608 \ln ^{4} \beta+\left(-\frac{150400}{7}+27648 \ln 2+\frac{256}{3} n_{f}\right) \ln ^{3} \beta\right. \\
&+\left(-315.57218-119.35529 n_{f}+496.30011 \frac{1}{\beta}\right) \ln ^{2} \beta+\left(3249.2403-19.935233 n_{f}-1.4285714 D_{Q \bar{Q}}^{(2)}\right. \\
&\left.\left.+\left(286.67132+6.8930570 n_{f}\right) \frac{1}{\beta}\right) \ln \beta+68.547138 \frac{1}{\beta^{2}}-\left(192.10086+0.96631115 n_{f}\right) \frac{1}{\beta}+C_{g g}^{(2)}\right] .
\end{aligned}
$$

Equations (12) and (14) are exact up to the unknown constant terms $C_{q \bar{q}}^{(2)}$ and $C_{g g}^{(2)}$ of order $\mathcal{O}\left(\beta^{0}\right)$, whereas Eq. (13) receives further corrections of order $\mathcal{O}\left(\beta^{3} \ln ^{2} \beta\right)$. Please note that the numerical coefficients in Eqs. (12) and (14) have slightly changed compared to Ref. [3]. The $\ln ^{2} \beta$ terms in Eqs. (12) and (14) are affected by using the exact coefficients (10) and (11) in the matching at NLO. The linear terms proportional to $\ln \beta$ in Eqs. (12) and (14) contain genuine two-loop contributions. Among those is the soft anomalous dimension $D_{Q \bar{Q}}^{(2)}$ (see Ref. [3]). Inserting the respective numerical coefficients we find $730.73916+$ $23.776275 n_{f}$ in Eq. (12) and $3035.5764-0.88761378 n_{f}$ in Eq. (14). The latter value has changed with respect to Ref. [3] due to a consistent separation of the color-singlet and color-octet contributions in $a_{0}^{g g}$ at NLO [17-19]. However the phenomenology is rather insensitive to this change and is only affected at the per mille level. The Coulomb terms $(\sim 1 / \beta)$ in Eqs. (12) and (14) contain all contributions from the two-loop virtual corrections. Equation (13) gives the leading (though formally power suppressed) contribution at two loops to the $g q$ channel. We include $f_{g q}^{(20)}$ in our analysis for three reasons. First, under evolution of the factorization scale the $g q$ channel mixes with the two other channels and for a consistent study of the factorization scale dependence this channel also needs to be taken into account. Next, the luminosity $L_{g q}$ in particular at LHC is sizable and Eq. (13) offers a way to control its numerical impact at higher orders. Finally, Eq. (13) provides a first step towards a general study of power suppressed but logarithmically enhanced terms near threshold for top-quark production.

In Eq. (1) the dependence of the scaling functions $f_{i j}$ on the renormalization and factorization scales, $\mu_{r}$ and $\mu_{f}$, respectively, can also be made explicit. Starting from the expansion in $\alpha_{s}$ through NNLO around $R=1$, i.e. $\mu_{r}=$ $\mu_{f}$, we introduce

$$
\begin{aligned}
f_{i j}(\rho, M, 1)= & f_{i j}^{(0)}(\rho)+4 \pi \alpha_{s}\left\{f_{i j}^{(10)}(\rho)+L_{M} f_{i j}^{(11)}(\rho)\right\} \\
& +\left(4 \pi \alpha_{s}\right)^{2}\left\{f_{i j}^{(20)}(\rho)+L_{M} f_{i j}^{(21)}(\rho)\right. \\
& \left.+L_{M}^{2} f_{i j}^{(22)}(\rho)\right\},
\end{aligned}
$$

where we abbreviate $L_{M}=\ln \left(\mu_{f}^{2} / m_{t}^{2}\right)$. The logarithmic tower in $M=\mu_{f}^{2} / m_{t}^{2}$, that is, all terms proportional to $L_{M}$ in Eq. (15), can be derived by renormalization group methods in a straightforward manner. The explicit results in the $\overline{\mathrm{MS}}$ scheme for all channels read at NLO

$$
\begin{gathered}
f_{i j}^{(11)}=-\left(2 P_{i j}^{(0)}-2 \beta_{0} \mathbb{1}\right) \otimes f_{i j}^{(0)}, \\
f_{g q}^{(11)}=-P_{g q}^{(0)} \otimes f_{g g}^{(0)}-\frac{1}{2 n_{f}} P_{q g}^{(0)} \otimes f_{q \bar{q}}^{(0)},
\end{gathered}
$$

where $i j=\{q \bar{q}, g g\}$ in Eq. (16). At NNLO we have

$$
\begin{aligned}
f_{q \bar{q}}^{(21)}=- & \left(2 P_{q q}^{(1)}-2 \beta_{1} \mathbb{1}\right) \otimes f_{q \bar{q}}^{(0)}-\left(2 P_{q q}^{(0)}-3 \beta_{0} \mathbb{1}\right) \otimes f_{q \bar{q}}^{(10)} \\
- & 2 P_{g q}^{(0)} \otimes f_{g q}^{(10)}, \\
f_{q \bar{q}}^{(22)}= & \left(2 P_{q q}^{(0)} \otimes P_{q q}^{(0)}-5 P_{q q}^{(0)} \beta_{0}+\frac{1}{2 n_{f}} P_{q g}^{(0)} \otimes P_{g q}^{(0)}\right. \\
& \left.+3 \beta_{0}^{2} \mathbb{1}\right) \otimes f_{q \bar{q}}^{(0)}+P_{g q}^{(0)} \otimes P_{g q}^{(0)} \otimes f_{g g}^{(0)} \\
f_{g q}^{(21)}=- & \frac{1}{2 n_{f}} P_{q g}^{(0)} \otimes f_{q \bar{q}}^{(10)}-\left(P_{q q}^{(0)}+P_{g g}^{(0)}-3 \beta_{0} \mathbb{1}\right) \otimes f_{g q}^{(10)} \\
- & P_{g q}^{(1)} \otimes f_{g g}^{(0)}-\frac{1}{2 n_{f}} P_{q g}^{(1)} \otimes f_{q \bar{q}}^{(0)}-P_{g q}^{(0)} \otimes f_{g g}^{(10)},
\end{aligned}
$$




$$
\begin{aligned}
f_{g q}^{(22)}= & \frac{1}{4 n_{f}} P_{q g}^{(0)} \otimes\left(3 P_{q q}^{(0)}+P_{g g}^{(0)}-5 \beta_{0} \mathbb{1}\right) \otimes f_{q \bar{q}}^{(0)} \\
& +\frac{1}{2} P_{g q}^{(0)} \otimes\left(P_{q q}^{(0)}+3 P_{g g}^{(0)}-5 \beta_{0} \mathbb{1}\right) \otimes f_{g g}^{(0)},
\end{aligned}
$$

$$
\begin{aligned}
f_{g g}^{(21)}= & -\left(2 P_{g g}^{(1)}-2 \beta_{1} \mathbb{1}\right) \otimes f_{g g}^{(0)}-\left(2 P_{g g}^{(0)}-3 \beta_{0} \mathbb{1}\right) \otimes f_{g g}^{(10)} \\
& -2 P_{q g}^{(0)} \otimes f_{g q}^{(10)},
\end{aligned}
$$

$$
\begin{aligned}
f_{g g}^{(22)}= & \frac{1}{2 n_{f}} P_{q g}^{(0)} \otimes P_{q g}^{(0)} \otimes f_{q \bar{q}}^{(0)}+\left(P_{q g}^{(0)} \otimes P_{g q}^{(0)}+2 P_{g g}^{(0)} \otimes P_{g g}^{(0)}\right. \\
& \left.-5 P_{g g}^{(0)} \beta_{0}+3 \beta_{0}^{2} \mathbb{1}\right) \otimes f_{g g}^{(0)} .
\end{aligned}
$$

In Eqs. (16)-(23) the $\otimes$ products have to be understood as standard convolutions and a sum over all active quarks and antiquarks is implied as well. The coefficients of the QCD $\beta$ function are given by

$$
\begin{aligned}
& \beta_{0}=\frac{1}{16 \pi^{2}}\left(11-\frac{2}{3} n_{f}\right), \\
& \beta_{1}=\frac{1}{\left(16 \pi^{2}\right)^{2}}\left(102-\frac{38}{3} n_{f}\right) .
\end{aligned}
$$

The splitting functions $P_{i j}^{(l)}$ can be taken e.g. from Refs. [28,29]. At leading order they read

$$
\begin{array}{r}
\left(16 \pi^{2}\right) P_{q q}^{(0)}(x)=\frac{4}{3}\left(\frac{4}{1-x}-2-2 x+3 \delta(1-x)\right) \\
\left(16 \pi^{2}\right) P_{q g}^{(0)}(x)=2 n_{f}\left(1-2 x+2 x^{2}\right) \\
\left(16 \pi^{2}\right) P_{g q}^{(0)}(x)=\frac{4}{3}\left(\frac{4}{x}-4+2 x\right) \\
\left(16 \pi^{2}\right) P_{g g}^{(0)}(x)=3\left(\frac{4}{1-x}+\frac{4}{x}-8+4 x-4 x^{2}\right. \\
\left.+\frac{11}{3} \delta(1-x)\right)-\frac{2}{3} n_{f} \delta(1-x)
\end{array}
$$

where the factor $\left(16 \pi^{2}\right)$ accounts for the normalization used in Eqs. (16)-(23). In general, we have $P_{i j}^{(l)}$ (this article $)=\left(16 \pi^{2}\right)^{-(l+1)} P_{i j}^{(l)}($ Refs. [28,29]). Please also note the explicit factor of $\left(2 n_{f}\right)^{-1}$ in Eqs. (17)-(23), which is due to the definition of $P_{q g}^{(0)}$ in Eq. (26) and $P_{q g}^{(1)}$ in Ref. [29]. Simple fully analytic expressions for $f_{q \bar{q}}^{(11)}, f_{g q}^{(11)}$ and $f_{g g}^{(11)}$ are long known [9] and precise fits for all scaling functions $f_{i j}^{(21)}, f_{i j}^{(22)}$ in Eqs. (18)-(23), typically to per mille accuracy, are presented in the appendix in Eqs. (A1)-(A6) and Tables V, VI, and VII. Finally, the complete scale dependence for $f_{i j}(\rho, M, R)$ in Eq. (1) with $\mu_{r} \neq \mu_{f}$ is easily obtained as

$$
\begin{aligned}
f_{i j}(\rho, M, R)= & f_{i j}(\rho, M, 1)+4 \pi \alpha_{s}\left\{2 \beta_{0} L_{R} f_{i j}^{(0)}\right\} \\
& +\left(4 \pi \alpha_{s}\right)^{2}\left\{3 \beta_{0} L_{R} f_{i j}^{(10)}+2 \beta_{1} L_{R} f_{i j}^{(0)}\right. \\
& \left.+3 \beta_{0} L_{R} L_{M} f_{i j}^{(11)}+3 \beta_{0}^{2} L_{R}^{2} f_{i j}^{(0)}\right\},
\end{aligned}
$$

$$
\begin{aligned}
f_{g q}(\rho, M, R)= & f_{g q}(\rho, M, 1)+\left(4 \pi \alpha_{s}\right)^{2}\left\{3 \beta_{0} L_{R} f_{g q}^{(10)}\right. \\
& \left.+3 \beta_{0} L_{M} f_{g q}^{(11)}\right\},
\end{aligned}
$$

where $L_{R}=\ln \left(\mu_{r}^{2} / \mu_{f}^{2}\right)$ and $i j=\{q \bar{q}, g g\}$ in Eq. (29).

\section{PHENOMENOLOGICAL APPLICATIONS}

We are now in the position to address the phenomenological consequences. The approximate NNLO prediction which includes exact dependence on all scales is based on Eqs. (12)-(14), (18)-(23), (29), and (30). If not otherwise stated, the top-quark mass is the pole mass at $m_{t}=$ $173 \mathrm{GeV}$.

Let us start with the scale dependence of the NNLO cross section as shown in Fig. 1. Our study of the theoretical uncertainty allows us to assess the effect of independent variations of the renormalization and factorization scale $\mu_{r}$ and $\mu_{f}$ in the scaling functions for the hard partonic scattering process in Eq. (1). In doing so, one should keep in mind, however, that all currently available PDF sets from global fits always fix $\mu_{r}=\mu_{f}$. We define the theory uncertainty arising from the independent variation of $\mu_{r}$ and $\mu_{f}$ in the standard range $\mu_{r}, \mu_{f} \in\left[m_{t} / 2,2 m_{t}\right]$ as

$$
\min \sigma\left(\mu_{r}, \mu_{f}\right) \leq \sigma\left(m_{t}\right) \leq \max \sigma\left(\mu_{r}, \mu_{f}\right) .
$$

The contour lines of the total cross section for the LHC and Tevatron arising from this procedure are shown in Fig. 1. The standard range $\mu_{r}, \mu_{f} \in\left[m_{t} / 2,2 m_{t}\right]$ corresponds to the region displayed in Fig. 1 because of $\log _{10}(2) \approx 0.3$. We have normalized all results to the value at $\mu_{r}=\mu_{f}=$ $m_{t}$ and the variation with fixed scales $\mu_{r}=\mu_{f}$ proceeds along the diagonal from the lower left to upper right in the plots. For Tevatron in Fig. 1(b) we see that the gradient in the complete $\left(\mu_{r}, \mu_{f}\right)$ plane is almost parallel to this diagonal, and thus the uncertainty according to Eq. (31) remains $-5 \% \leq \Delta \sigma \leq+3 \%$ in very good agreement with previous results $[3,4]$. At LHC in Fig. 1(a) the maximal deviations in the $\left(\mu_{r}, \mu_{f}\right)$ plane are $-3 \%$ located at $\left(2 m_{t}, 2 m_{t}\right)$ and $+1 \%$ at roughly $\left(m_{t} / 2,2 m_{t}\right)$, so that the uncertainty range (31) becomes slightly larger, $-3 \% \leq$ $\Delta \sigma \leq+1 \%$ compared to what has been derived before with a fixed scale ratio $\mu_{r}=\mu_{f}$. Very similar numbers for both colliders are obtained with the PDF set CTEQ6.6 [7]. Also recall that we include the $g q$ channel through two loops. Thanks to Eqs. (18)-(23) we control the exact scale dependence also for all contributions proportional to the parton luminosity $L_{g q}$. We conclude from Fig. 1 that the 


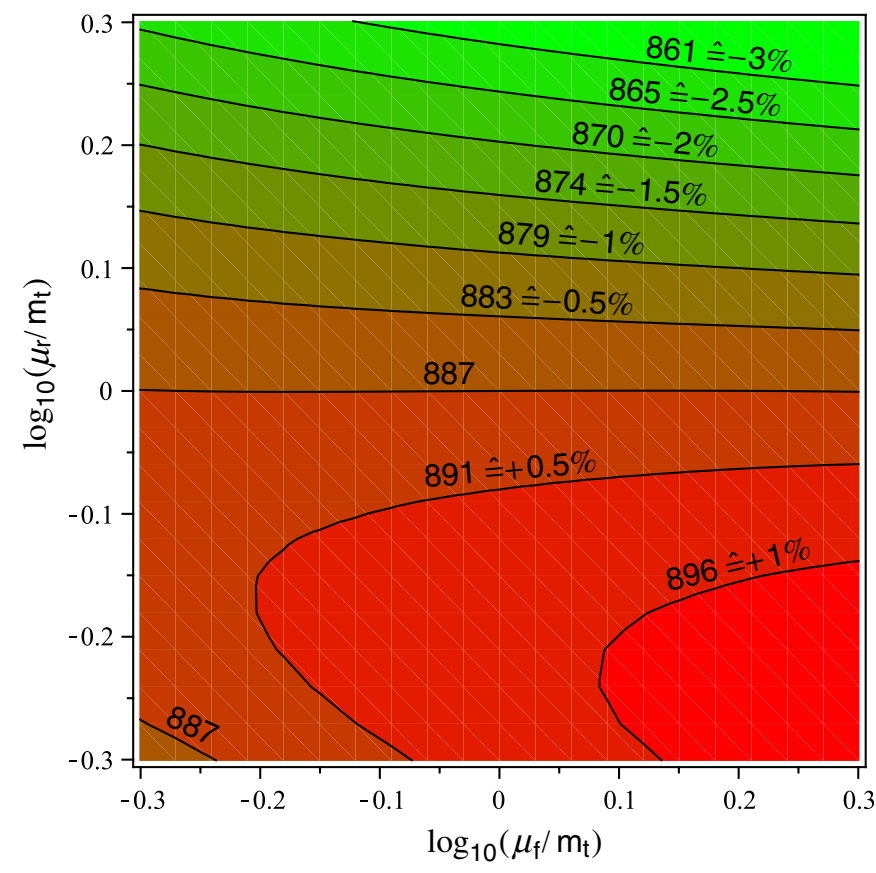

(a)

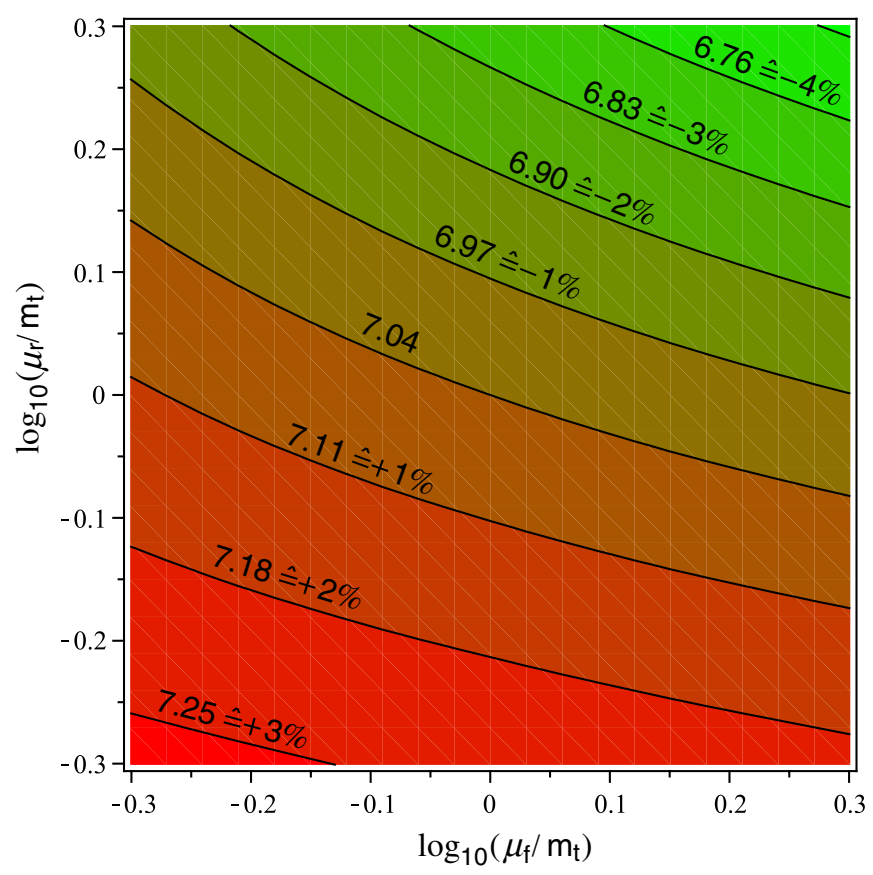

(b)

FIG. 1 (color online). The contour lines of the total hadronic cross section from the independent variation of renormalization and factorization scale $\mu_{r}$ and $\mu_{f}$ for LHC with $\sqrt{S}=14 \mathrm{TeV}$ (left) and Tevatron with $\sqrt{S}=1.96 \mathrm{TeV}$ (right) with MSTW 2008 [14]. The cross sections are normalized to the values at $\mu_{r}=\mu_{f}=m_{t}$ and the range corresponds to $\mu_{r}, \mu_{f} \in\left[m_{t} / 2,2 m_{t}\right]$.

theoretical uncertainty due to $\mu_{r}$ and $\mu_{f}$ variation is well estimated by the case of identical scales $\mu_{r}=\mu_{f}$.

In order to quantify the PDF uncertainty we apply the standard definition

$$
\Delta \sigma=\frac{1}{2} \sqrt{\sum_{k=1, n_{\mathrm{PDF}}}\left(\sigma_{k+}-\sigma_{k-}\right)^{2}}
$$

which determines $\Delta \sigma$ from the variations $\sigma_{k \pm}$ of the cross section with respect to the $k$ parameters of the PDF fit. Typically the PDF error is added linearly to the theory uncertainty obtained from the scale variation. This is the commonly adopted choice and we employ the PDF sets CTEQ6.6 [7] and MSTW 2008 [14]. The latter set gives two uncertainties at different confidence levels (C.L.), one at $68 \%$ C.L. and the second at $90 \%$ C.L. Throughout this study we use $68 \%$ C.L. only. Moreover in the chosen interval $\mu \in\left[m_{t} / 2,2 m_{t}\right]$ for a given $\mu=\mu_{r}=\mu_{f}$ the error $\Delta \sigma(\mu)$ in Eq. (32) has only a very weak scale dependence. That is to say we find to good accuracy $\Delta \sigma\left(\mu=m_{t} / 2\right) \simeq \Delta \sigma\left(\mu=m_{t}\right) \simeq \Delta \sigma\left(\mu=2 m_{t}\right)$ so that a determination of $\Delta \sigma$ at the central scale $\mu=m_{t}$ should suffice for all practical purposes.

In Fig. 2 we show the mass dependence of the total cross section, comparing the NLO and our approximate NNLO prediction. The band summarizes the total theoretical uncertainty from the linear combination of the scale uncertainty for the case $\mu_{r}=\mu_{f}$ and the PDF uncertainty
Eq. (32). We display the LHC and Tevatron predictions using the MSTW 2008 PDF set [14]. The improvement of the NNLO prediction is manifest for both colliders.

Next, we discuss the sources of remaining systematical uncertainties. Undoubtedly, a complete calculation of the complete NNLO QCD corrections to hadronic top-quark pair production would be highly desirable (see Refs. [3037] for progress in this direction). This lacking, the main systematic uncertainty of our approximate NNLO result are the subleading terms in the scaling function $f_{i j}^{(20)}$. They might become accessible by extending the approach of Refs. [26,27] or else could be modeled through power suppressed terms in Mellin space (see e.g. the scheme $A=$ 2 in Ref. [38]). By including the leading term for $f_{g q}^{(20)}$ we have taken a first step in this direction and we have found numerically small effects only. In order to quantify our systematical uncertainty, we adopt the following prescription: We compute the ratio $\sigma_{\mathrm{NLL}+\mathrm{Coul}} / \sigma_{\text {exact }}$ at one loop, where $\sigma_{\mathrm{NLL}+\text { Coul }}$ only contains the Sudakov logarithms and the Coulomb terms in $f_{q \bar{q}}^{(10)}$ and $f_{g g}^{(10)}$, i.e. the content of the square brackets in Eqs. (7) and (9). This checks how well the exact hadronic cross section in Eq. (1) is approximated, if only the threshold approximation enters in the convolution with the parton luminosities. Typically we find $\sigma_{\mathrm{NLL}+\text { Coul }} / \sigma_{\text {exact }} \gtrsim 0.7(0.9)$ for the LHC (Tevatron). If translated to the genuine two-loop contribution (see e.g. Table I below), then a systematic uncertainty of $\mathcal{O}(30 \%)$ implies a cross section uncertainty of $\Delta \sigma \simeq \mathcal{O}(15) \mathrm{pb}$ at 


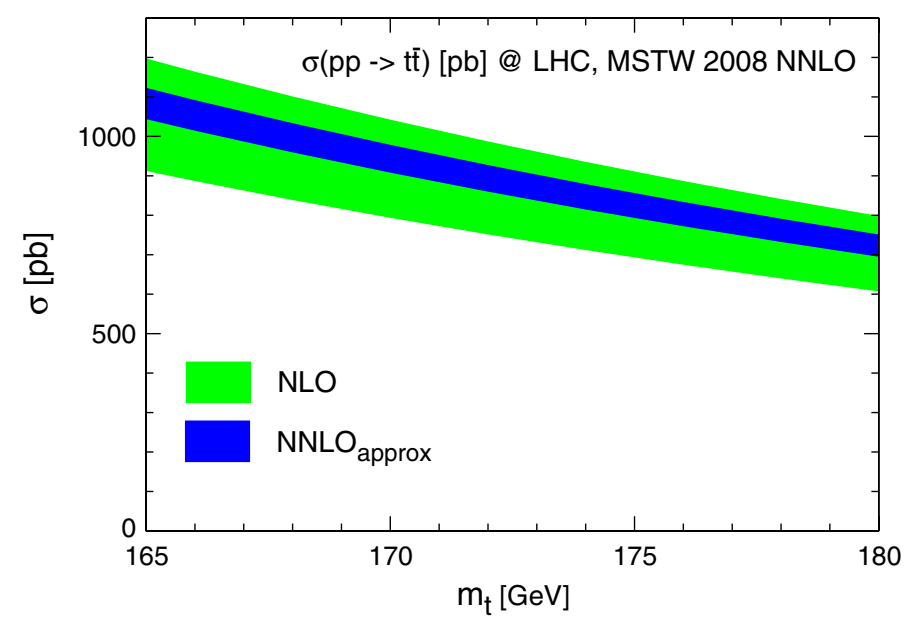

(a)

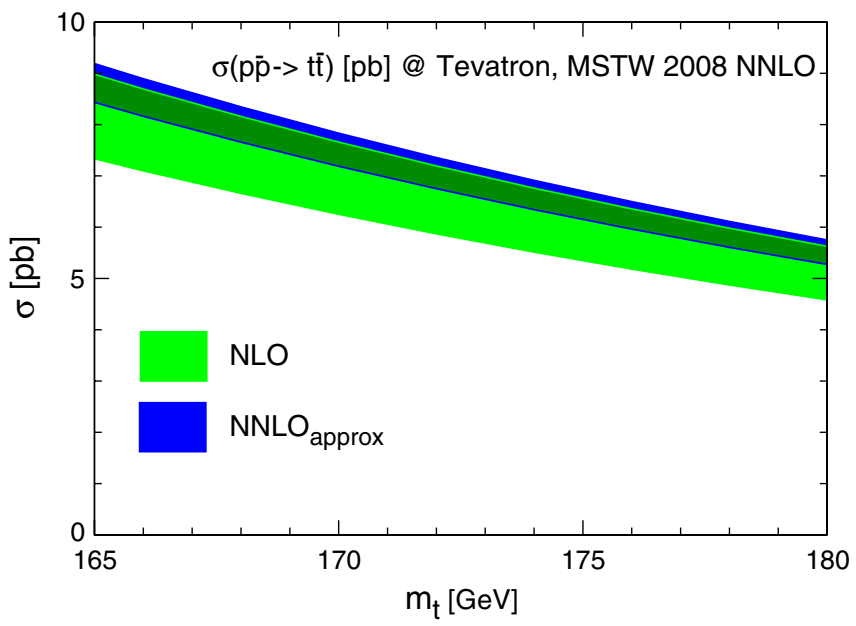

(b)

FIG. 2 (color online). The mass dependence of the total cross section at NLO (green) and approximate NNLO (blue) order for LHC at $\sqrt{S}=14 \mathrm{TeV}$ (left) and Tevatron at $\sqrt{S}=1.96 \mathrm{TeV}$ (right) and the PDF set MSTW 2008 [14]. The bands denote the theoretical uncertainty from scale variation keeping $\mu_{r}=\mu_{f}$ and the PDF uncertainty in the range $\left[m_{t} / 2,2 m_{t}\right]$. In (b) for the Tevatron the NLO and approximate NNLO bands overlap only partially giving rise to the corresponding medium band (dark green).

LHC and of $\Delta \sigma \simeq \mathcal{O}(0.2) \mathrm{pb}$ at Tevatron. These numbers are corroborated by other observations, like the generally small impact of the $g q$ channel which is entirely subleading.

How does this affect the previous discussion of the scale dependence? Let us define a systematic uncertainty $\Delta_{\text {sys }}$ obtained from a variation of the scaling functions $f_{i j}^{(20)}$ in Eqs. (12)-(14) by $\pm 30 \%$. All other scaling functions are known exactly anyway. The result for our NNLO cross section (always normalized to the value at $\mu=m_{t}$ and $\Delta_{\text {sys }}=0$ ) is shown in Fig. 3 for $\mu=\mu_{r}=\mu_{f}$ and the MSTW 2008 PDF set [14]. It is obvious that the predictions are very stable within the standard range $\mu \in\left[m_{t} / 2,2 m_{t}\right]$ for all cases, i.e. $\Delta_{\text {sys }}=0$ and $\sigma \pm \Delta_{\text {sys }}$. For the case $\Delta_{\text {sys }}=0$ we find a variation of $-3 \% \leq \Delta \sigma \leq+0.5 \%$ for LHC and $-5 \% \leq \Delta \sigma \leq+3 \%$ for Tevatron (compatible with Fig. 1) and similar numbers for the other two cases, $\sigma \pm \Delta_{\text {sys }}$.

TABLE I. The LO, NLO and approximate NNLO prediction for the total cross section at LHC $(\sqrt{S}=14 \mathrm{TeV})$ and Tevatron $(\sqrt{S}=1.96 \mathrm{TeV})$ using $m_{t}=171 \mathrm{GeV}$, the PDF set CTEQ6.6 [7] and $\mu_{r}=\mu_{f}=m_{t}$. For comparison we also give the previous numbers of Refs. [3,4].

\begin{tabular}{lcc}
\hline \hline & LHC & Tevatron \\
\hline$\sigma_{\mathrm{LO}}[\mathrm{pb}]$ & 583.7 & 5.820 \\
$\sigma_{\mathrm{NLO}}[\mathrm{pb}]$ & 877.4 & 7.229 \\
$\sigma_{\mathrm{NNLO}}[\mathrm{pb}]$ (this work) & 923.0 & 7.814 \\
$\sigma_{\mathrm{NNLO}}[\mathrm{pb}]($ Refs. [3,4]) & 920.5 & 7.810 \\
\hline \hline
\end{tabular}

In the present analysis, we have also neglected the effect of the new parton channels $q q, \bar{q} \bar{q}$ and $q_{i} \bar{q}_{j}$ (for unlike flavors $i \neq j$ ), which come in through real emission at NNLO only. Important insight can be gained here from the recent calculation $[39,40]$ of the radiative corrections for $t \bar{t}+1$-jet production at NLO, because they represent a significant part of the complete NNLO corrections for inclusive top-quark pair production. At NLO $t \bar{t}+1$-jet production contains the one-loop one-parton real emissions as well as the double real emission processes, and the latter also include the above mentioned new channels. It was found that the radiative corrections at the scale $\mu_{r}=$ $\mu_{f}=m_{t}$ are rather small. Depending on the kinematical cuts (e.g. on the transverse momentum of the jet) they amount to $\mathcal{O}(20) \mathrm{pb}$ at $\mathrm{LHC}$ and to $\mathcal{O}(0.2) \mathrm{pb}$ at Tevatron (see Ref. [40]). This provides further evidence that the hard corrections to the inclusive top-quark pair production at NNLO are indeed not large and it supports the estimate of our systematical uncertainty.

To summarize, our approximate NNLO prediction leaves us with a rather small residual theoretical uncertainty based on the scale variation. It is also worth stressing that the numerical impact of our theory improvements in Eqs. (12)-(14) and (18)-(23) is rather small, which again nicely illustrates the stability of the approximate NNLO predictions. In Table I we compare with our previous numbers [3,4] for the CTEQ6.6 [7] set at $m_{t}=171 \mathrm{GeV}$ and $\mu_{r}=\mu_{f}=m_{t}$. At Tevatron, we find no changes, as the cross section is entirely dominated by the $q \bar{q}$ channel at parton kinematics close to threshold. At LHC, there is a small net change of $2.5 \mathrm{pb}$ in the prediction. Here, the effect of the improved NLO matching [in particular, the 


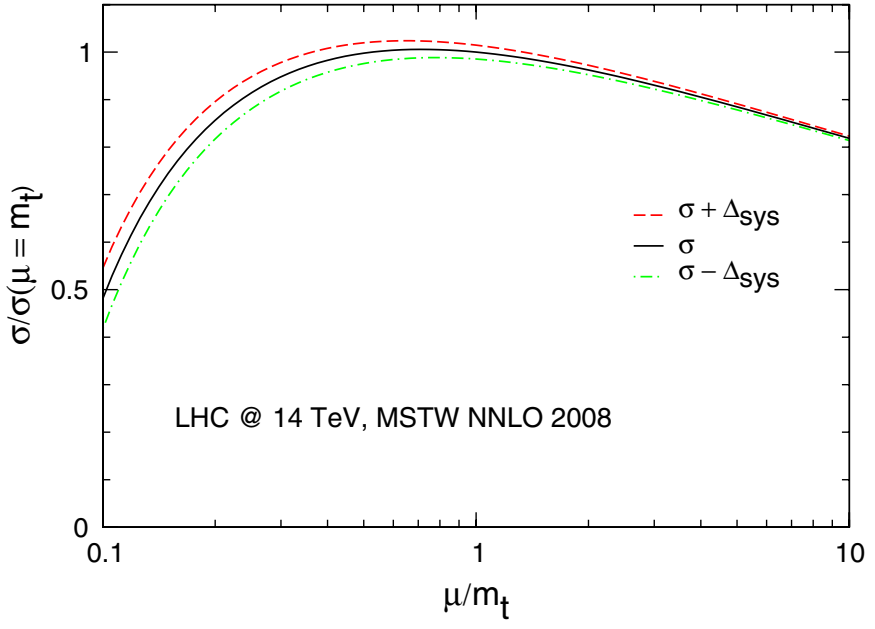

(a)

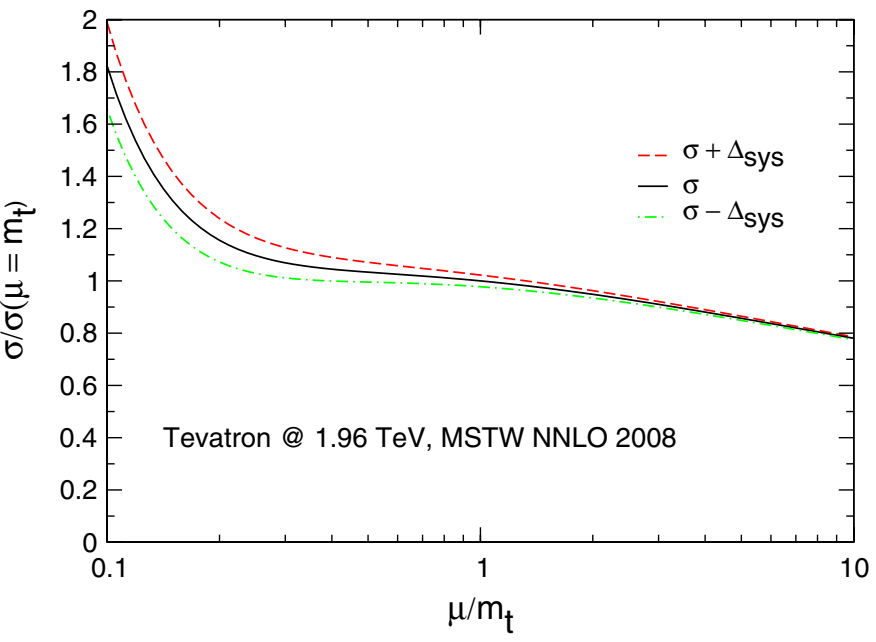

(b)

FIG. 3 (color online). The scale dependence of the approximate NNLO cross section $\sigma_{\mathrm{NNLO}}$ for the choice $\mu=\mu_{r}=\mu_{f}$ using the MSTW 2008 PDF set [14] at LHC with $\sqrt{S}=14 \mathrm{TeV}$ (left) and Tevatron with $\sqrt{S}=1.96 \mathrm{TeV}$ (right). $\Delta_{\text {sys }}$ denotes the estimated systematic uncertainty of our threshold approximation at NNLO and all results are normalized to the value of $\sigma\left(\mu=m_{t}\right)$.

$\ln ^{2} \beta$ term in Eq. (14) depending on the constant $a_{0}^{g g}$ in Eq. (11)] and the leading two-loop term $f_{g q}^{(20)}$ in Eq. (13) in the $g q$ channel partially compensate. The numerical impact of the exact color decomposition at NLO [affecting the $\ln \beta$ term in Eq. (14)] is completely negligible.

As a central result of the current studies we quote our approximate NNLO prediction at $\operatorname{LHC}(\sqrt{S}=14 \mathrm{TeV})$ and Tevatron $(\sqrt{S}=1.96 \mathrm{TeV})$ for a pole mass of $m_{t}=$ $173 \mathrm{GeV}$. For the MSTW 2008 set [14] we have

$$
\sigma_{\text {LHC }}=887 \mathrm{pb} \quad{ }_{-33}^{+9} \mathrm{pb}(\text { scale }) \quad{ }_{-15}^{+15} \mathrm{pb}(\text { MSTW 2008), }
$$

$$
\begin{aligned}
& \sigma_{\mathrm{Tev}}=7.04 \mathrm{pb} \quad{ }_{-0.36}^{+0.24} \mathrm{pb}(\text { scale }) \\
& +0.14 \mathrm{pb}(\mathrm{MSTW} 2008),
\end{aligned}
$$

and for CTEQ6.6 [7],

$$
\begin{array}{ccc}
\sigma_{\mathrm{LHC}}=874 \mathrm{pb} & { }_{-33}^{+9} \mathrm{pb}(\text { scale }) & { }_{-28}^{+28} \mathrm{pb}(\mathrm{CTEQ6.6}), \\
\sigma_{\mathrm{Tev}}=7.34 \mathrm{pb} & \stackrel{+0.38}{+0.24} \mathrm{pb}(\text { scale }) & { }_{-0.41}^{+0.41} \mathrm{pb} \text { (CTEQ6.6) }
\end{array}
$$

Please note that the MSTW 2008 set [14] is based on a global analysis to NNLO in QCD while CTEQ6.6 [7] performs a fit to NLO only. Therefore, the two PDF sets return slightly different default values for the coupling constant $\alpha_{s}$. While the LHC predictions of both sets are largely in agreement for these choices of $\alpha_{s}$, the difference in the Tevatron predictions can be attributed to differences in the parametrization of the light quark PDFs at large $x$. In addition, there is a systematical uncertainty in Eqs. (33)-
(36) estimated to be $\mathcal{O}(2 \%)$ due to unknown NNLO contributions, i.e. the exact expression for $f_{i j}^{(20)}$ in Eqs. (12)(14). One could of course argue that the accuracy of a given PDF set should match the accuracy of the theoretical prediction of the partonic cross section. However, we would like to disentangle the shift originating from corrections to the hard parton scattering (which is the main subject of our paper) from PDF effects. Therefore we always use the same order in perturbation theory as far as the chosen PDFs are concerned (cf. Table I and also Appendix B of Ref. [3]).

For applications, the mass dependence of the hadronic cross section (1) is conveniently parametrized by the following simple fit formula:

$$
\sigma\left(m_{t}, \mu\right)=a+b x+c x^{2}+d x^{3}+e x^{4}+f x^{5}+g x^{6},
$$

with $\mu=\mu_{r}=\mu_{f}, x=\left(m_{t} / \mathrm{GeV}-173\right)$ and the scale choices $\mu=m_{t}, 2 m_{t}, m_{t} / 2$. For the cross section at LHC $(\sqrt{S}=14 \mathrm{TeV}$ and $\sqrt{S}=10 \mathrm{TeV})$ and Tevatron $(\sqrt{S}=$ $1.96 \mathrm{TeV}$ ) all fit coefficients are listed in Tables III and IV,

TABLE II. The LO, NLO and approximate NNLO results for the top-quark mass in the $\overline{\mathrm{MS}}$ scheme $(\bar{m})$ and the pole mass scheme $\left(m_{t}\right)$ for the measured cross section of $\sigma=8.18 \mathrm{pb}$ at Tevatron [8]. The uncertainties in the table reflect the quoted experimental uncertainties.

\begin{tabular}{lcc}
\hline \hline & $\bar{m}[\mathrm{GeV}]$ & $m_{t}[\mathrm{GeV}]$ \\
\hline LO & $159.2_{-3.4}^{+3.5}$ & $159.2_{-3.4}^{+3.5}$ \\
NLO & $159.8_{-3.3}^{+3.3}$ & $165.8_{-3.5}^{+3.5}$ \\
NNLO & $160.0_{-3.2}^{+3.3}$ & $168.2_{-3.5}^{+3.6}$ \\
\hline \hline
\end{tabular}


TABLE III. Fit coefficients to Eq. (37) for $\sigma\left(\mu=m_{t}, 2 m_{t}, m_{t} / 2\right)$ and $\sigma\left(\mu=m_{t}\right) \pm \Delta \sigma$ for the PDF set CTEQ6.6 [7] and the colliders LHC and Tevatron.

\begin{tabular}{|c|c|c|c|c|c|c|c|}
\hline & $a[\mathrm{pb}]$ & $b[\mathrm{pb}]$ & {$[\mathrm{pb}]$} & $d[\mathrm{pb}]$ & $e[\mathrm{pb}]$ & $f[\mathrm{pb}]$ & $g[\mathrm{pb}]$ \\
\hline \multicolumn{8}{|c|}{ LHC $\sqrt{s}=14 \mathrm{TeV}, \mathrm{CTEQ} 6.6$} \\
\hline$\sigma\left(\mu=m_{t}\right)$ & $8.74428 \times 10^{2}$ & $\times 10^{1}$ & $3.74083>$ & $-4.62418 \times 10^{-3}$ & $4.99329 \times 10^{-5}$ & -4.5546 & 2.370 \\
\hline$\sigma\left(\mu=m_{t} / 2\right)$ & $8.72517 \times 10^{2}$ & $-2.34260 \times 10^{1}$ & $3.72103 \times 10^{-1}$ & $-4.59525 \times 10^{-3}$ & $4.95790 \times 10^{-5}$ & $10^{-7}$ & 2.347 \\
\hline$\sigma\left(\mu=2 m_{t}\right)$ & $8.41176 \times 10^{2}$ & $-2.26414 \times 10^{1}$ & $3.60329 \times 10^{-1}$ & $-4.45624 \times 1$ & $4.81468 \times 10^{-5}$ & $-4.39515 \times$ & 2.289 \\
\hline$\sigma\left(\mu=m_{t}\right)+$ & $9.02378 \times 10^{2}$ & $-2.40942 \times 10^{1}$ & $3.81862 \times 10^{-1}$ & $-4.71806 \times 10^{-3}$ & $5.10568 \times$ & $-4.67281 \times$ & 2.438 \\
\hline$\sigma\left(\mu=m_{t}\right)-\Delta \sigma$ & $8.46479 \times 10^{2}$ & -2.2944 & 3.6629 & $-4.53009 \times 10^{-3}$ & $4.88109 \times 10^{-5}$ & -4.4375 & 2.3036 \\
\hline \multicolumn{8}{|c|}{ LHC $\sqrt{s}=10 \mathrm{TeV}$, CTEQ6.6 } \\
\hline$\sigma\left(\mu=m_{t}\right)$ & $3.96877 \times 10^{2}$ & -1.120 & -1 & $10^{-3}$ & -5 & $0^{-7}$ & 1. \\
\hline$\sigma\left(\mu=m_{t} / 2\right)$ & $3.97124 \times 10^{2}$ & -1.118 & $1.84706 \times 10^{-1}$ & $-2.35501 \times 10^{-3}$ & $2.61183 \times 10^{-5}$ & -2.429 & 1.278 \\
\hline$\sigma\left(\mu=2 m_{t}\right)$ & $3.79852 \times 10^{2}$ & $-1.07358 \times 10^{1}$ & $1.77667 \times 10^{-1}$ & $-2.26977 \times 10^{-3}$ & $2.52223 \times 10^{-5}$ & -2.352 & 1.240 \\
\hline$\sigma(\mu=n$ & $4.15125 \times 10^{2}$ & $-1.15947 \times 10^{1}$ & $1.90285 \times 10^{-1}$ & $-2.41772 \times 10^{-3}$ & $2.67843 \times 10^{-5}$ & $-2.49480 \times 10^{-7}$ & $1.31488 \times 10^{-9}$ \\
\hline$\sigma\left(\mu=m_{t}\right)-\Delta \sigma$ & $3.78628 \times 10^{2}$ & $-1.08207 \times 10^{1}$ & $1.80416 \times 10^{-1}$ & $-2.31532 \times 10^{-3}$ & $2.57769 \times 10^{-5}$ & $\times 10^{-7}$ & $1.26569 \times 10^{-9}$ \\
\hline \multicolumn{8}{|c|}{ Tevatron $\sqrt{s}=1.96 \mathrm{TeV}$, CTEQ6.6 } \\
\hline$\sigma\left(\mu=m_{t}\right)$ & $7.34317 \times 10^{0}$ & -2 & 3.94086 & -5.2230 & $\times 10^{-7}$ & $-5.99414 \times 1$ & 3.27 \\
\hline$\sigma(\mu=$, & $7.58312 \times 10^{0}$ & $-2.34571 \times 10^{-1}$ & $4.05822 \times 10^{-3}$ & $-5.37018 \times 10^{-5}$ & $.25408 \times 10^{-7}$ & $-6.13901 \times 10^{-9}$ & $3.35467 \times 10^{-11}$ \\
\hline$\sigma\left(\mu=2 m_{t}\right)$ & $\times 10^{0}$ & $-2.15748 \times 10^{-1}$ & $3.73128 \times 10^{-3}$ & $-4.93012 \times 10^{-5}$ & $.73218 \times 10^{-7}$ & $-5.62092 \times 10^{-9}$ & $3.07038 \times$ \\
\hline$\sigma\left(\mu=m_{t}\right)+$ & $7.75854 \times 10^{0}$ & $-2.42254 \times 10^{-1}$ & $4.23665 \times 10^{-3}$ & $-5.65955 \times 10^{-5}$ & $6.63296 \times 10^{-7}$ & $-6.52935 \times 10^{-9}$ & $3.57062 \times 10^{-11}$ \\
\hline$\sigma(\mu=1$ & $6.92780 \times 10^{0}$ & $-2.12718 \times 10$ & $3.64506 \times 10^{-3}$ & $-4.78628 \times 10^{-5}$ & $5.55679 \times 10^{-7}$ & $-5.46023 \times 10^{-9}$ & $2.99003 \times 10^{-11}$ \\
\hline
\end{tabular}

where we have used the PDF sets CTEQ6.6 [7] and MSTW 2008 [14]. In the mass range $150 \mathrm{GeV} \leq m_{t} \leq 220 \mathrm{GeV}$, the accuracy of the fit is always better than $2.5 \%$.

Let us briefly mention also other types of radiative corrections, which have not been considered here, e.g. in Eqs. (33)-(37). Within QCD these are bound state effects for the $t \bar{t}$ pair near threshold [18,19]. They affect the total cross section at LHC of the order $\mathcal{O}(10) \mathrm{pb}$ and, even more so, differential distributions in the invariant mass of the top-quark pair. At Tevatron, due to the dominance of the $q \bar{q}$ channel in the color-octet configuration, they are negligible though. Precision analyses at the percent level naturally need to consider also the electroweak radiative corrections at NLO [20-22]. Depending on the Higgs mass they cause a decrease relative to the LO cross section between $\mathcal{O}(2 \%)$ for a light Higgs $\left(m_{h}=120 \mathrm{GeV}\right)$ and $\mathcal{O}(2.5 \%)$ for a heavy Higgs $\left(m_{h}=1000 \mathrm{GeV}\right)$ at the LHC. This amounts to a negative contribution $\Delta \sigma_{\mathrm{EW}} \simeq \mathcal{O}(10-15) \mathrm{pb}$. At the Tevatron, the electroweak radiative corrections are almost zero for a light Higgs $\left(m_{h}=120 \mathrm{GeV}\right)$ and give a negative contribution of order $\mathcal{O}(1 \%)$, i.e. $\Delta \sigma_{\mathrm{EW}} \simeq \mathcal{O}(0.05) \mathrm{pb}$ for a heavy Higgs $\left(m_{h}=1000 \mathrm{GeV}\right)$.

TABLE IV. Same as Table III for the PDF set MSTW2008 [14] at NNLO. The PDF uncertainty $\Delta \sigma$ has been obtained with the $68 \%$ confidence level set.

\begin{tabular}{|c|c|c|c|c|c|c|c|}
\hline & $a[\mathrm{pb}]$ & $b[\mathrm{pb}]$ & $c[\mathrm{pb}]$ & $d[\mathrm{pb}]$ & $e[\mathrm{pb}]$ & $f[\mathrm{pb}]$ & $g[\mathrm{pb}]$ \\
\hline \multicolumn{8}{|c|}{ LHC $\sqrt{s}=14 \mathrm{TeV}$, MSTW $2008 \mathrm{NNLO}$} \\
\hline$\sigma\left(\mu=m_{t}\right)$ & $8.87496 \times 10^{2}$ & $-2.38344 \times 10^{1}$ & $3.78224 \times 10^{-1}$ & $-4.66307 \times 10^{-3}$ & $5.02155 \times 10^{-5}$ & $-4.56910 \times 10^{-7}$ & $2.37374 \times 10^{-9}$ \\
\hline$\sigma\left(\mu=m_{t} / 2\right)$ & $8.85530 \times 10^{2}$ & $-2.37387 \times 10^{1}$ & $3.76203 \times 10^{-1}$ & $-4.63331 \times 10^{-3}$ & $4.98411 \times 10^{-5}$ & $-4.52980 \times 10^{-7}$ & $2.35137 \times 10^{-9}$ \\
\hline$\sigma\left(\mu=2 m_{t}\right)$ & $8.54052 \times 10^{2}$ & $-2.29566 \times 10^{1}$ & $3.64547 \times 10^{-1}$ & $-4.49661 \times 10^{-3}$ & $4.84539 \times 10^{-5}$ & $-4.41574 \times 10^{-7}$ & $2.29907 \times 10^{-9}$ \\
\hline$\sigma\left(\mu=m_{t}\right)+\Delta \sigma$ & $9.02902 \times 10^{2}$ & $-2.41907 \times 10^{1}$ & $3.83190 \times 10^{-1}$ & $-4.71808 \times 10^{-3}$ & $5.07642 \times 10^{-5}$ & $-4.61804 \times 10^{-7}$ & $2.39989 \times 10^{-9}$ \\
\hline$\sigma\left(\mu=m_{t}\right)-\Delta \sigma$ & $8.72090 \times 10^{2}$ & $-2.34783 \times 10^{1}$ & $3.73257 \times 10^{-1}$ & $-4.60776 \times 10^{-3}$ & $4.96661 \times 10^{-5}$ & $-4.52297 \times 10^{-7}$ & $2.35168 \times 10^{-9}$ \\
\hline \multicolumn{8}{|c|}{ LHC $\sqrt{s}=10 \mathrm{TeV}$, MSTW $2008 \mathrm{NNLO}$} \\
\hline$\sigma\left(\mu=m_{t}\right)$ & $4.03219 \times 10^{2}$ & $-1.13904 \times 10^{1}$ & $1.88177 \times 10^{-1}$ & $-2.39835 \times 10^{-3}$ & $2.65811 \times 10^{-5}$ & $-2.47337 \times 10^{-7}$ & $1.30217 \times 10^{-9}$ \\
\hline$\sigma\left(\mu=m_{t} / 2\right)$ & $4.03439 \times 10^{2}$ & $-1.13695 \times 10^{1}$ & $1.87488 \times 10^{-1}$ & $-2.38625 \times 10^{-3}$ & $2.64067 \times 10^{-5}$ & $-2.45191 \times 10^{-7}$ & $1.28831 \times 10^{-9}$ \\
\hline$\sigma\left(\mu=2 m_{t}\right)$ & $3.86012 \times 10^{2}$ & $-1.09154 \times 10^{1}$ & $1.80486 \times 10^{-1}$ & $-2.30194 \times 10^{-3}$ & $2.55275 \times 10^{-5}$ & $-2.37710 \times 10^{-7}$ & $1.25272 \times 10^{-9}$ \\
\hline$\sigma\left(\mu=m_{t}\right)+\Delta \sigma$ & $4.11912 \times 10^{2}$ & $-1.16047 \times 10^{1}$ & $1.91287 \times 10^{-1}$ & $-2.43344 \times 10^{-3}$ & $2.69297 \times 10^{-5}$ & $-2.50369 \times 10^{-7}$ & $1.31793 \times 10^{-9}$ \\
\hline$\sigma\left(\mu=m_{t}\right)-\Delta \sigma$ & $3.94526 \times 10^{2}$ & $-1.11761 \times 10^{1}$ & $1.85066 \times 10^{-1}$ & $-2.36310 \times 10^{-3}$ & $2.62315 \times 10^{-5}$ & $-2.44419 \times 10^{-7}$ & $1.28819 \times 10^{-9}$ \\
\hline \multicolumn{8}{|c|}{ Tevatron $\sqrt{s}=1.96 \mathrm{TeV}$, MSTW $2008 \mathrm{NNLO}$} \\
\hline$\sigma\left(\mu=m_{t}\right)$ & $7.04217 \times 10^{0}$ & $-2.18800 \times 10^{-1}$ & $3.80366 \times 10^{-3}$ & $-5.06795 \times 10^{-5}$ & $5.96308 \times 10^{-7}$ & $-5.92150 \times 10^{-9}$ & $3.26369 \times 10^{-11}$ \\
\hline$\sigma\left(\mu=m_{t} / 2\right)$ & $7.27746 \times 10^{0}$ & $-2.25794 \times 10^{-1}$ & $3.92060 \times 10^{-3}$ & $-5.21816 \times 10^{-5}$ & $6.12661 \times 10^{-7}$ & $-6.05762 \times 10^{-9}$ & $3.32360 \times 10^{-11}$ \\
\hline$\sigma\left(\mu=2 m_{t}\right)$ & $6.67970 \times 10^{0}$ & $-2.07517 \times 10^{-1}$ & $3.60070 \times 10^{-3}$ & $-4.78294 \times 10^{-5}$ & $5.60141 \times 10^{-7}$ & $-5.52680 \times 10^{-9}$ & $3.02883 \times 10^{-11}$ \\
\hline$\sigma\left(\mu=m_{t}\right)+\Delta \sigma$ & $7.18407 \times 10^{0}$ & $-2.22429 \times 10^{-1}$ & $3.85513 \times 10^{-3}$ & $-5.13171 \times 10^{-5}$ & $6.05482 \times 10^{-7}$ & $-6.04397 \times 10^{-9}$ & $3.34549 \times 10^{-11}$ \\
\hline$\sigma\left(\mu=m_{t}\right)-\Delta \sigma$ & $6.90028 \times 10^{0}$ & $-2.15171 \times 10^{-1}$ & $3.75214 \times 10^{-3}$ & $-5.00394 \times 10^{-5}$ & $5.87170 \times 10^{-7}$ & $-5.80214 \times 10^{-9}$ & $3.18553 \times 10^{-11}$ \\
\hline
\end{tabular}




\section{THE TOP-QUARK MASS IN THE MS SCHEME}

So far we have used the pole mass of the top quark as a definition of the mass parameter. However, it is well known that the concept of the pole mass has intrinsic theoretical limitations owing to the fact that the top quark is a colored object. As such it does not appear as an asymptotic state of the $S$ matrix due to confinement. In other words the $S$ matrix does not have a pole in the top-quark channel. The impact of different mass renormalizations has been investigated in great detail in the context of top-quark mass measurements at a future linear collider where a precision of the order of a few hundred $\mathrm{MeV}$ is envisaged. In particular, it has been shown that indeed the conceptual limitations of the pole mass lead to a poorly behaved perturbative series. A class of alternative mass definitions, so-called short distance masses, offer a solution to this problem, e.g. the $1 S$ mass or the potential subtracted mass (see e.g. Ref. [41]).

In the following we study the impact of the conversion from the pole mass scheme to the $\overline{\mathrm{MS}}$ scheme (see Refs. [23-25], and references therein) for the total cross section of top-quark hadroproduction. This is a novel feature and, in principle, the cross section in terms of the $\overline{\mathrm{MS}}$ mass can be used for a direct measurement of the running mass at a high scale. This is similar to the case of $b$-quark production at LEP (see Refs. [42-47]). Let us first describe briefly how we translate the predictions for the total cross section from the pole mass to the $\overline{\mathrm{MS}}$ mass scheme. The starting point is the well-known relation between the pole mass $m_{t}$ and the $\overline{\mathrm{MS}}$ mass $m\left(\mu_{r}\right)$ to NNLO:

$$
m_{t}=m\left(\mu_{r}\right)\left(1+a_{s}\left(\mu_{r}\right) d^{(1)}+a_{s}\left(\mu_{r}\right)^{2} d^{(2)}\right),
$$

with $a_{s}=\alpha_{s}^{\left(n_{f}=5\right)} / \pi$ (i.e. five active flavors) and coefficients $d^{(i)}$, which in general depend on the ratio $\mu_{r}^{2} / m\left(\mu_{r}\right)^{2}$

$$
\begin{gathered}
d^{(1)}=\frac{4}{3}+L_{m\left(\mu_{r}\right)}, \\
d^{(2)=} \frac{307}{32}+2 \zeta_{2}+\frac{2}{3} \zeta_{2} \ln 2-\frac{1}{6} \zeta_{3}+\frac{509}{72} L_{m\left(\mu_{r}\right)} \\
+\frac{47}{24} L_{m\left(\mu_{r}\right)}^{2}-\left(\frac{71}{144}+\frac{1}{3} \zeta_{2}+\frac{13}{36} L_{m\left(\mu_{r}\right)}\right. \\
\left.+\frac{1}{12} L_{m\left(\mu_{r}\right)}^{2}\right) n_{f}+\frac{4}{3} \sum_{i} \Delta\left(m_{i} / m_{t}\right) .
\end{gathered}
$$

Here $n_{f}$ denotes the number of light flavors and $L_{m\left(\mu_{r}\right)}=$ $\ln \left(\mu_{r}^{2} / m\left(\mu_{r}\right)^{2}\right)$. The function $\Delta\left(m_{i} / m_{t}\right)$ accounts for all massive quarks $m_{i}$ lighter than the top quark. For all light quarks we set $m_{i}=0$ so the sum in Eq. (40) vanishes. Note also that the decoupling of the top quark is assumed to be done at the scale of the $\overline{\mathrm{MS}}$ mass $m\left(\mu_{r}\right)$.
Let us start by making the mass dependence in the total cross section manifest order by order in perturbation theory. For the pole mass $m_{t}$ we have through NNLO

$$
\sigma=a_{s}^{2} \sum_{i=0}^{2} a_{s}^{i} \sigma^{(i)}\left(m_{t}\right)
$$

Next, we use the relation (38) above to convert from the pole mass to the $\overline{\mathrm{MS}}$ mass $m(m)$. For simplicity we abbreviate $\bar{m}=m(m)$ and obtain

$$
\begin{aligned}
\sigma= & a_{s}^{2} \sum_{i=0}^{2} a_{s}^{i}\left(\sigma^{(i)}(\bar{m})+\left.\bar{m} \sum_{l=1}^{i} d^{(l)} \partial_{m} \sigma^{(i-l)}(m)\right|_{m=\bar{m}}\right. \\
& \left.+\left.\delta_{i, 2} \frac{1}{2}\left(\bar{m} d^{(1)}\right)^{2} \partial_{m}^{2} \sigma^{(0)}(m)\right|_{m=\bar{m}}\right) .
\end{aligned}
$$

We note that the coefficients $d^{(i)}$ have to be evaluated for $\mu_{r}=\bar{m}$ (corresponding to the scale of $\alpha_{s}$ ). Thus, the task in Eq. (42) amounts to determine the derivatives of the cross sections $\sigma^{(i)}$ with respect to the mass. To do so in practice we have chosen the following approach. For all coefficients $\sigma^{(i)}$ we use the ansatz of Eq. (47) to parametrize the mass dependence. More precisely we evaluate the hadronic cross section order by order in perturbation theory for a fixed renormalization and factorization scale. Then, varying the top-quark mass (in the pole mass scheme) and performing a fit similar to what has been discussed before in Eq. (37) we obtain the total cross section in the following form:

$$
\sigma=a_{s}^{2} \sum_{i=0}^{2} a_{s}^{i} \sum_{k=0}^{N}\left(m_{t}-m_{0}\right)^{k} c_{k}^{(i)},
$$

where $c_{k}^{(i)}$ denote the (order-dependent) fit coefficients. $N$ is the order of the polynomial in $m_{t}[N=6$ in Eq. (37)] and $m_{0}$ is our fixed reference mass [taken to be $173 \mathrm{GeV}$ in Eq. (37)]. Since all dependence on the pole mass $m_{t}$ is manifest, it is now a straightforward exercise to convert to the $\overline{\mathrm{MS}}$ mass $\bar{m}$ and to perform the derivatives in Eq. (42),

$$
\begin{aligned}
\sigma= & a_{s}^{2} \sum_{i=0}^{2} a_{s}^{i} \sum_{k=0}^{N}\left(\left(\bar{m}-m_{0}\right)^{k} c_{k}^{(i)}+k \bar{m}\left(\bar{m}-m_{0}\right)^{k-1}\right. \\
& \times \sum_{l=1}^{i} d^{(l)} c_{k}^{(i-l)}+\delta_{i, 2} \frac{1}{2} k(k-1) \\
& \left.\times \bar{m}^{2}\left(\bar{m}-m_{0}\right)^{k-2}\left(d^{(1)}\right)^{2} c_{k}^{(0)}\right) \\
\stackrel{m_{0}=}{=} & \bar{m} \\
& a_{s}^{2}\left(c_{0}^{(0)}+a_{s}\left\{c_{0}^{(1)}+\bar{m} d^{(1)} c_{1}^{(0)}\right\}+a_{s}^{2}\left\{c_{0}^{(2)}+\bar{m} d^{(1)} c_{1}^{(1)}\right.\right. \\
& \left.\left.+\bar{m} d^{(2)} c_{1}^{(0)}+\bar{m}^{2}\left(d^{(1)}\right)^{2} c_{2}^{(0)}\right\}\right) .
\end{aligned}
$$

If the expansion point $m_{0}$ is chosen to be the $\overline{\mathrm{MS}}$ mass $\bar{m}$, Eq. (44) simplifies considerably and the truncation of the power series in $\bar{m}$ to first (second) order is exact at NLO (NNLO). Generally though, for applications, it is of some advantage to keep $m_{0}$ at a fixed numerical value and to rely 
on the fact that our ansatz (43) with a polynomial of high enough degree $N$ approximates all coefficients $\sigma^{(i)}$ and their first two derivatives sufficiently well. As discussed below Eq. (37), the choice $N=6$ achieves per mille accuracy in the phenomenologically interesting range. We have also checked that the choices $m_{0}=\bar{m}$ and $m_{0} \neq \bar{m}$ yield the same result.

We stress again that we have fixed $\mu_{r}=\bar{m}$ in Eq. (44). However, it is also possible to restore the complete renormalization scale dependence using the well-known relation for the running coupling

$$
\begin{aligned}
a_{s}(\bar{m})= & a_{s}\left(\mu_{r}\right)\left(1+4 \pi^{2} a_{s}\left(\mu_{r}\right) L_{\bar{R}} \beta_{0}\right. \\
& +\left(4 \pi^{2}\right)^{2} a_{s}\left(\mu_{r}\right)^{2}\left(\beta_{1} L_{\bar{R}}+\beta_{0}^{2} L_{\bar{R}}^{2}\right),
\end{aligned}
$$

with $L_{\bar{R}}=\ln \left(\mu_{r}^{2} / \bar{m}^{2}\right)$ and $\beta_{0}$ and $\beta_{1}$ given in Eq. (24). To summarize, Eq. (44) represents an explicit expression for the total cross section of top-quark hadroproduction with the top-quark mass defined in the $\overline{\mathrm{MS}}$ scheme.

Let us illustrate the phenomenological consequences of the $\overline{\mathrm{MS}}$ mass for predictions at Tevatron and LHC. In Fig. 4 we plot the scale dependence of the total cross section again at the various orders in perturbation theory. The value of $\bar{m}=163 \mathrm{GeV}$ roughly corresponds to a pole mass of $m_{t}=173 \mathrm{GeV}$ and we choose three (fixed) values for the factorization scale $\mu_{f}=\bar{m} / 2, \bar{m}$ and $2 \bar{m}$. The band to the left denotes the maximum and the minimum values of $\mu_{r} \in[\bar{m} / 2,2 \bar{m}]$ for the three choices of $\mu_{f}$ according to Eq. (31); cf. the contour plot in Fig. 1 for the pole mass. We observe a great stability with respect to scale variations when including higher order perturbative corrections

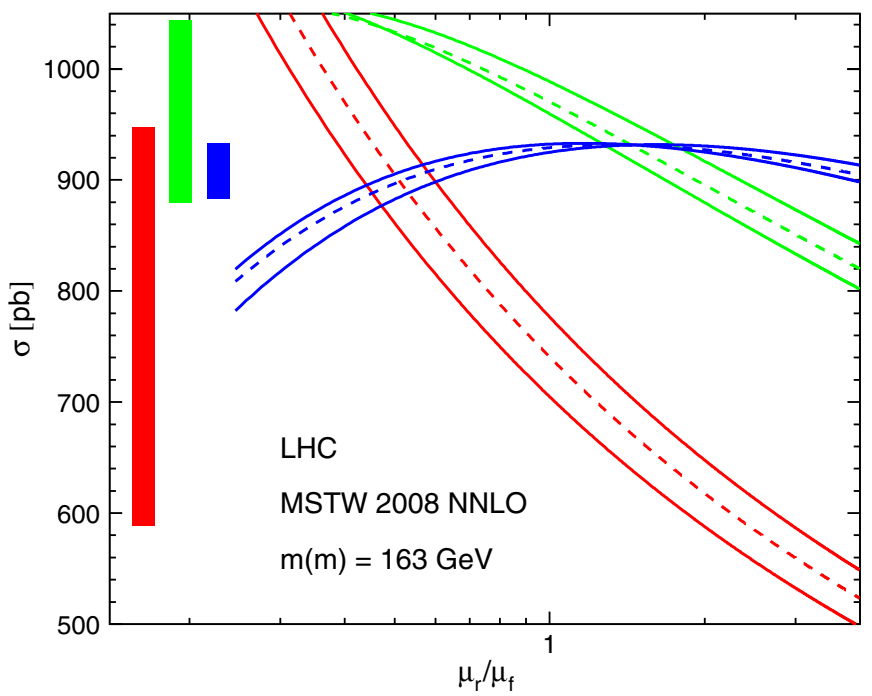

(a) through NNLO. Remarkably, at Tevatron, the scale variation at NNLO is even reduced further by more than a factor of 2 compared to the result in the pole mass scheme.

Next, in Fig. 5 we show the mass dependence of the total cross section employing the $\overline{\mathrm{MS}}$ mass definition and performing the same scale variation as above, i.e. $\mu_{f}=\bar{m} / 2$, $\bar{m}$ and $2 \bar{m}$ and $\mu_{r} \in[\bar{m} / 2,2 \bar{m}]$. Upon adding the higher order perturbative corrections we observe as a striking feature the extremely small numerical effect of the radiative corrections. For example, for $\bar{m}=163 \mathrm{GeV}$ at Tevatron, we find the effect of the NLO corrections to be only $1.5 \%$ and even less $(0.9 \%)$ for the approximate NNLO results. Also for the LHC, we observe a much faster convergence of the perturbative expansion when using the $\overline{\mathrm{MS}}$ mass. The NLO (approximate NNLO) corrections amount to $31 \%$ (4\%) at $\bar{m}=163 \mathrm{GeV}$ which is roughly half of the size of the corrections in the pole mass scheme. This demonstrates an extremely good stability of the perturbative series in the $\overline{\mathrm{MS}}$ mass scheme. We can understand this behavior qualitatively by looking at the mass dependence of the scaling functions in Eqs. (4)-(9). We find e.g. $\partial_{m} f_{i j}^{(0)} \simeq\left(1-\beta^{2}\right) / \beta$, which implies sizably enhanced contributions near partonic threshold, i.e. precisely in the region which contributes dominantly in the convolution with the parton luminosities; cf. Eq. (1). This observation is yet another argument in favor of the phenomenological importance of our approximate NNLO predictions in Eqs. (12)-(14).

A different way to address the issue of perturbative stability is the extraction of the $\overline{\mathrm{MS}}$ mass from the total

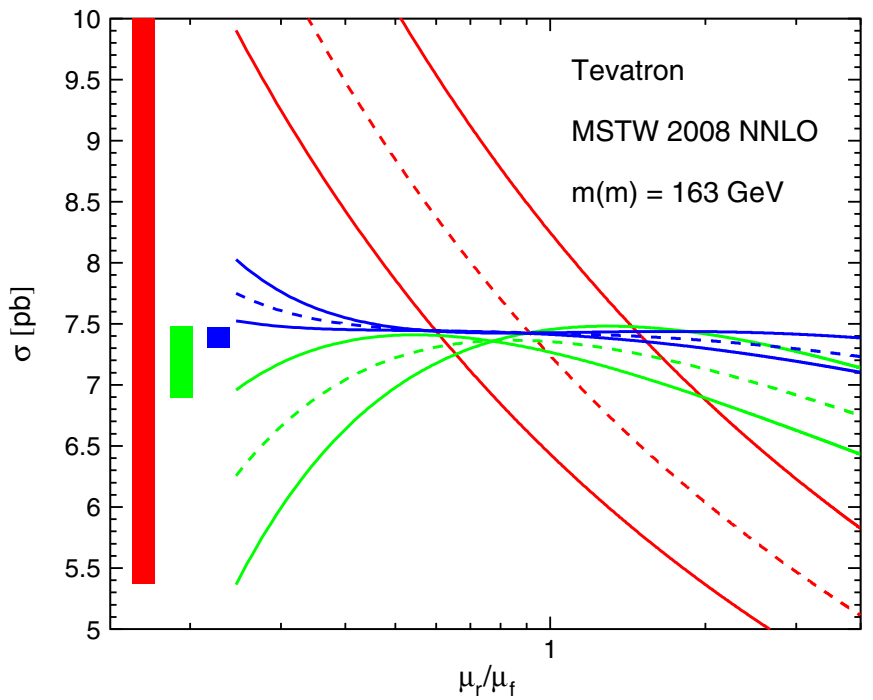

(b)

FIG. 4 (color online). The scale dependence of the total cross section with the top-quark mass in the $\overline{\mathrm{MS}}$ scheme at $\bar{m}=163 \mathrm{GeV}$ at LO (red), NLO (green) and approximate NNLO (blue). The dashed lines denote the $\mu_{f}=\bar{m}$ for the factorization scale, the solid lines the maximal deviations for $\mu_{r} \in[\bar{m} / 2,2 \bar{m}]$ and $\mu_{f}=\bar{m} / 2, \bar{m}$ and $2 \bar{m}$. We use the MSTW 2008 PDF set [14] at LHC with $\sqrt{S}=$ $14 \mathrm{TeV}$ (left) and Tevatron with $\sqrt{S}=1.96 \mathrm{TeV}$ (right). The vertical bars indicate the size of the scale variation in the standard range $[\bar{m} / 2,2 \bar{m}]$. 


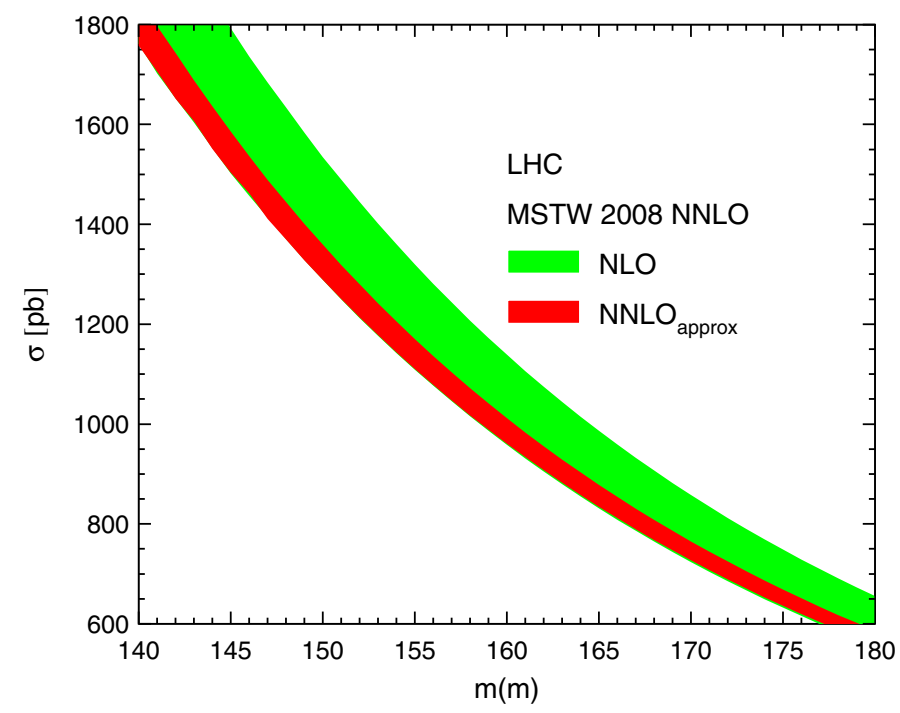

(a)

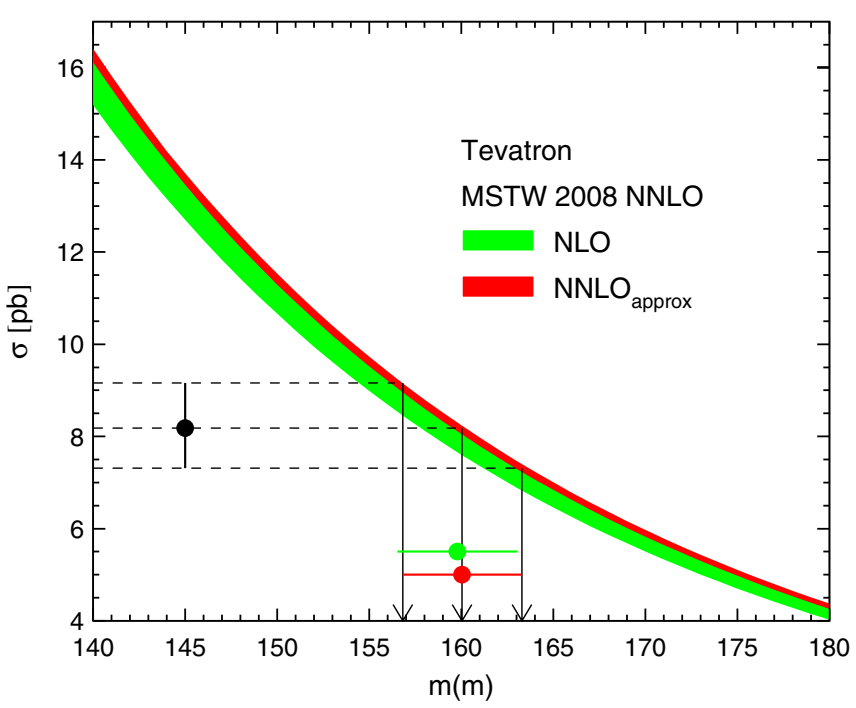

(b)

FIG. 5 (color online). The mass dependence of the total cross section for the $\overline{\mathrm{MS}}$ mass $\bar{m}$ at NLO (green) and approximate NNLO (red) order with the scale variation in the range $\mu_{r} \in[\bar{m} / 2,2 \bar{m}]$ and $\mu_{f}=\bar{m} / 2, \bar{m}$ and $2 \bar{m}$ for the MSTW 2008 PDF set [14] at LHC with $\sqrt{S}=14 \mathrm{TeV}$ (left) and Tevatron with $\sqrt{S}=1.96 \mathrm{TeV}$ (right). The value for the Tevatron cross section is taken from Ref. [8].

cross section as measured at Tevatron. Reference [8] quotes a value with a combined uncertainty of $\sigma=$ $8.18_{-0.87}^{+0.98} \mathrm{pb}$ for a top-quark mass $m_{t}=170 \mathrm{GeV}$ along with a (weak) dependence on the value of the mass, e.g. $\sigma=7.99 \mathrm{pb}$ for the latest world average [13], $m_{t}=$ $173.1_{-1.3}^{+1.3} \mathrm{GeV}$. Using the measured value of $\sigma=$ $8.18 \mathrm{pb}$ which is consistent with the theory predictions of Refs. [3,4] (and with this work; see Table II) we extract the $\overline{\mathrm{MS}}$ mass $\bar{m}$ order by order. As mentioned earlier we use the same NNLO PDF set of MSTW 2008 [14] independent of the order of perturbation theory and the results at LO, NLO and approximate NNLO are given in Table II. The value of $\bar{m}=160.0_{-3.2}^{+3.3} \mathrm{GeV}$ represents to the best of our knowledge the first direct determination of the running top-quark mass from experimental data. For comparison, we also quote the values of the pole mass $m_{t}$ at the respective order extracted in the same way by directly comparing the theory prediction with the measured cross section. Alternatively, we can also convert the $\overline{\mathrm{MS}}$ mass value back to the pole mass scheme with the help of Eq. (38). Our NNLO value for $\bar{m}$ corresponds to $m_{t}=168.9_{-3.4}^{+3.5} \mathrm{GeV}$, which constitutes a theoretically well-defined determination of the pole mass and is also (within the experimental uncertainties) in agreement with the world average [13] of $m_{t}=$ $173.1_{-1.3}^{+1.3} \mathrm{GeV}$. To summarize, the $\overline{\mathrm{MS}}$ mass scheme is distinguished by the great stability in the value of the extracted top-quark mass. This feature has been studied in the past in detail for processes at a future linear collider [41] and our observation is also in agreement with recent considerations based on the renormalization group flow for heavy quark masses [48].

\section{SUMMARY}

In this Letter, we update and extend the predictions of Refs. [3,4] for the cross section of top-quark hadroproduction at LHC and Tevatron. We have applied some improvements in the threshold approximation for the two-loop scaling functions (12)-(14) as described in the text. We provide new and precise parametrizations in Eqs. (7)-(9) and (18)-(23) for all scaling functions that can be determined exactly. All fit functions are documented in the appendix. Moreover, we have performed the independent variation of the renormalization and the factorization scale with the help of Eqs. (29) and (30). As a novel aspect, in addition to the conventionally used pole mass we provide predictions for the total cross section employing the $\overline{\mathrm{MS}}$ definition for the mass parameter. The central result is Eq. (44).

Our main phenomenological results are the parametrizations in Eq. (37), Tables III and IV and the cross sections in Eqs. (33)-(36) for the pole mass. The differences with respect to our previous numbers are quite small, though; see Table I. The theory uncertainty according to Eq. (31) defined by exploring the $\left(\mu_{r}, \mu_{f}\right)$ plane in the standard range $\mu_{r}, \mu_{f} \in\left[m_{t} / 2,2 m_{t}\right]$ does not differ significantly from the case of fixed scales $\mu_{r}=\mu_{f}$. We have also addressed the residual systematical uncertainty due to the threshold approximation and we have quantified the effect of other higher order corrections, such as electroweak or QCD bound state effects. The most interesting aspect of our phenomenological studies consists of the conversion to the $\overline{\mathrm{MS}}$ mass scheme in Figs. 4 and 5. The cross section predictions with the $\overline{\mathrm{MS}}$ mass definition exhibit a greatly 
improved pattern of apparent convergence for the perturbative expansion and very good stability with respect to scale variations. This leads to very stable values for the extracted mass parameter $\bar{m}$ as given in Table II. In particular, we find

$$
\bar{m}=160.0_{-3.2}^{+3.3} \mathrm{GeV} \text {. }
$$

This is the first direct determination of the running topquark mass from top-quark pair production. The corresponding value for the pole mass derived from Eq. (46) reads

$$
m_{t}=168.9_{-3.4}^{+3.5} \mathrm{GeV},
$$

which is consistent with the current world average [13], $m_{t}=173.1_{-1.3}^{+1.3} \mathrm{GeV}$. Altogether, this provides substantial support in view of the reliability of our approximate NNLO numbers. We believe that the QCD radiative corrections for top-quark pair production at hadron colliders are well under control.

\section{ACKNOWLEDGMENTS}

We would like to thank W. Bernreuther, M. Cacciari, A. Hoang and H. Kawamura for stimulating discussions and A. Mitov for his very lively contributions at LoopFest VIII and the CERN Theory Institute TOPO9. This work is supported by the Helmholtz Gemeinschaft under Contract No. VH-NG-105 and by the Deutsche Forschungsgemeinschaft under Contract No. SFB/TR 9. P.U. acknowledges the support of the Initiative and Networking Fund of the Helmholtz Gemeinschaft, Contract No. HA-101 ("Physics at the Terascale").

\section{APPENDIX: USEFUL FORMULAS}

$$
\begin{aligned}
f_{q \bar{q}}^{(21)}= & \frac{1}{\left(16 \pi^{2}\right)^{2}} f_{q \bar{q}}^{(0)}\left[-\frac{8192}{9} \ln ^{3} \beta+\left(\frac{12928}{3}-\frac{32768}{9} \ln 2\right) \ln ^{2} \beta+\left(-840.51065+70.183854 \frac{1}{\beta}\right) \ln \beta-82.246703 \frac{1}{\beta}\right. \\
& +467.90402]+\frac{n_{f}}{\left(16 \pi^{2}\right)^{2}} f_{q \bar{q}}^{(0)}\left[-\frac{256}{3} \ln ^{2} \beta+\left(\frac{2608}{9}-\frac{2816}{9} \ln 2\right) \ln \beta+6.5797363 \frac{1}{\beta}-64.614276\right] \\
& +h\left(\beta, b_{i}+n_{f} c_{i}\right)-\frac{4 n_{f}^{2}}{\left(16 \pi^{2}\right)^{2}} f_{q \bar{q}}^{(0)}\left[\frac{4}{3} \ln 2-\frac{2}{3} \ln \rho-\frac{10}{9}\right]
\end{aligned}
$$

$$
\begin{aligned}
f_{q \bar{q}}^{(22)}= & \frac{1}{\left(16 \pi^{2}\right)^{2}} f_{q \bar{q}}^{(0)}\left[\frac{2048}{9} \ln ^{2} \beta+\left(-\frac{7840}{9}+\frac{4096}{9} \ln 2\right) \ln \beta+270.89724\right]+\frac{n_{f}}{\left(16 \pi^{2}\right)^{2}} f_{q \bar{q}}^{(0)}\left[\frac{320}{9} \ln \beta-\frac{596}{9}+\frac{320}{9} \ln 2\right] \\
& +h\left(\beta, b_{i}+n_{f} c_{i}\right)+\frac{4 n_{f}^{2}}{3\left(16 \pi^{2}\right)^{2}} f_{q \bar{q}}^{(0)}
\end{aligned}
$$

$$
\begin{aligned}
f_{g q}^{(21)}= & -\frac{\pi}{\left(16 \pi^{2}\right)^{2}} \beta^{3}\left[\frac{770}{27} \ln ^{2} \beta+\left(-\frac{6805}{81}+\frac{6160}{81} \ln 2\right) \ln \beta+0.13707784 \frac{1}{\beta}+0.22068868\right] \\
& -\frac{\pi n_{f}}{81\left(16 \pi^{2}\right)^{2}} \beta^{3}\left[46 \ln \beta-\frac{163}{3}+76 \ln 2\right]+h_{g q}^{(b)}\left(\beta, b_{i}+n_{f} c_{i}\right),
\end{aligned}
$$

$$
f_{g q}^{(22)}=\frac{\pi}{\left(16 \pi^{2}\right)^{2}} \beta^{3}\left[\frac{385}{81} \ln \beta-\frac{1540}{243}+\frac{385}{81} \ln 2\right]+h_{g q}^{(b)}\left(\beta, b_{i}+n_{f} c_{i}\right),
$$

$$
\begin{aligned}
f_{g g}^{(21)}= & \frac{1}{\left(16 \pi^{2}\right)^{2}} f_{g g}^{(0)}\left[-4608 \ln ^{3} \beta+\left(\frac{109920}{7}-18432 \ln 2\right) \ln ^{2} \beta+\left(69.647185-248.15005 \frac{1}{\beta}\right) \ln \beta+56.867721 \frac{1}{\beta}\right. \\
& +17.010070]+\frac{n_{f}}{\left(16 \pi^{2}\right)^{2}} f_{g g}^{(0)}\left[-64 \ln ^{2} \beta+\left(\frac{4048}{21}-192 \ln 2\right) \ln \beta-3.4465285 \frac{1}{\beta}-37.602004\right] \\
& +h\left(\beta, b_{i}+n_{f} c_{i}\right),
\end{aligned}
$$


TABLE V. Coefficients for fits of the $q \bar{q}$ scaling functions.

\begin{tabular}{|c|c|c|c|c|c|}
\hline \multirow[b]{2}{*}{$i$} & \multirow{2}{*}{$\begin{array}{c}f_{q \bar{q}}^{(10)} \\
a_{i}\end{array}$} & \multicolumn{2}{|c|}{$f_{q \bar{q}}^{(21)}$} & \multicolumn{2}{|c|}{$f_{q \bar{q}}^{(22)}$} \\
\hline & & $b_{i}$ & $c_{i}$ & $b_{i}$ & $c_{i}$ \\
\hline 1 & 0.07120603 & -0.15388765 & -0.07960658 & 0.37947056 & -0.00224114 \\
\hline 2 & -1.27169999 & 4.85226571 & 0.50111294 & -4.25138041 & 0.02685576 \\
\hline 3 & 1.24099536 & -7.06602840 & -0.09496432 & 2.91716094 & -0.01777126 \\
\hline 4 & -0.04050443 & 2.36935255 & -0.32590203 & 0.94994470 & -0.00626121 \\
\hline 5 & 0.02053737 & -0.03634651 & -0.02229012 & 0.10537529 & -0.00062062 \\
\hline 6 & -0.31763337 & 1.25860837 & 0.23397666 & -1.69689874 & 0.00980999 \\
\hline 7 & -0.71439686 & 2.75441901 & 0.30223487 & -2.60977181 & 0.01631175 \\
\hline 8 & 0.01170002 & -1.26571709 & 0.13113818 & -0.27215567 & 0.00182500 \\
\hline 9 & 0.00148918 & -0.00230536 & -0.00162603 & 0.00787855 & -0.00004627 \\
\hline 10 & -0.14451497 & 0.15633927 & 0.08378465 & -0.47933827 & 0.00286176 \\
\hline 11 & -0.13906364 & 1.79535231 & -0.09147804 & -0.18217132 & 0.00111459 \\
\hline 12 & 0.01076756 & 0.36960437 & -0.01581518 & -0.04067972 & 0.00017425 \\
\hline 13 & 0.49397845 & -5.45794874 & 0.26834309 & 0.54147194 & -0.00359593 \\
\hline 14 & -0.00567381 & -0.76651636 & 0.03251642 & 0.08404406 & -0.00035339 \\
\hline 15 & -0.53741901 & 5.35350436 & -0.25679483 & -0.51918414 & 0.00363300 \\
\hline 16 & -0.00509378 & 0.39690927 & -0.01670122 & -0.04336452 & 0.00017915 \\
\hline 17 & 0.18250366 & -1.68935685 & 0.07993054 & 0.15957988 & -0.00115164 \\
\hline
\end{tabular}

$$
\begin{aligned}
f_{g g}^{(22)}= & \frac{1}{\left(16 \pi^{2}\right)^{2}} f_{g g}^{(0)}\left[1152 \ln ^{2} \beta+(-2568+2304 \ln 2) \ln \beta\right. \\
& -79.74312140]+\frac{n_{f}}{\left(16 \pi^{2}\right)^{2}} f_{g g}^{(0)}[16 \ln \beta-16 \\
& +16 \ln 2]+h\left(\beta, b_{i}+n_{f} c_{i}\right),
\end{aligned}
$$

where all threshold logarithms $\ln (\beta)$ and the Coulomb corrections $(\sim 1 / \beta)$ are exact. The fit functions are given in Eqs. (A7)-(A9) and all parameters of the fit are listed in Tables V, VI, and VII. The fits to the scaling functions $f_{i j}^{(21)}$, $f_{i j}^{(22)}$ in Eqs. (A1)-(A6) are, in general, accurate at the per mille level. Exceptions are regions close to zero, which is not surprising. There we retain an accuracy better than $1 \%$.

FORTRAN subroutines with the parametrizations of all scaling functions and the coefficient in Tables V, VI, and

\begin{tabular}{|c|c|c|c|c|c|}
\hline \multirow[b]{2}{*}{$i$} & \multirow{2}{*}{$\begin{array}{c}f_{g q}^{(10)} \\
a_{i} \\
\end{array}$} & \multicolumn{2}{|c|}{$f_{g q}^{(21)}$} & \multicolumn{2}{|c|}{$f_{g q}^{(22)}$} \\
\hline & & $b_{i}$ & $c_{i}$ & $b_{i}$ & $c_{i}$ \\
\hline 1 & -0.26103970 & -0.00120532 & 0.00003257 & -0.00022247 & 0.00001789 \\
\hline 2 & 0.30192672 & -0.04906353 & 0.00014276 & 0.00050422 & 0.00000071 \\
\hline 3 & -0.01505487 & -0.20885725 & -0.00402017 & -0.02945504 & -0.00020581 \\
\hline 4 & -0.00142150 & -13.73137224 & 0.06329831 & 0.34340412 & 0.00108759 \\
\hline 5 & -0.04660699 & 14.01818840 & -0.05952825 & -0.31894917 & -0.00086284 \\
\hline 6 & -0.15089038 & -0.00930488 & 0.00002694 & 0.00009213 & 0.00000010 \\
\hline 7 & -0.25397761 & -0.52223668 & 0.00159804 & 0.00690402 & 0.00001638 \\
\hline 8 & -0.00999129 & -4.68440515 & 0.01522672 & 0.07847233 & 0.00022730 \\
\hline 9 & 0.39878717 & -7.61046166 & 0.02869438 & 0.16042051 & 0.00045698 \\
\hline 10 & -0.02444172 & 1.36687743 & -0.00875589 & -0.05186974 & -0.00025620 \\
\hline 11 & -0.14178346 & 1.84698291 & -0.00800271 & -0.03861021 & -0.00016026 \\
\hline 12 & 0.01867287 & -7.26265988 & 0.04043479 & 0.21650362 & 0.00070713 \\
\hline 13 & 0.00238656 & -4.89364026 & 0.01965878 & 0.10137656 & 0.00034937 \\
\hline 14 & -0.00003399 & 11.04566784 & -0.05262293 & -0.28056264 & -0.00072547 \\
\hline 15 & -0.00000089 & 4.13660190 & -0.01457395 & -0.08090469 & -0.00025525 \\
\hline 16 & 0.00000000 & -6.33477051 & 0.02314616 & 0.13077889 & 0.00034015 \\
\hline 17 & 0.00000000 & -1.08995440 & 0.00291792 & 0.01813862 & 0.00006613 \\
\hline 18 & 0.00000000 & 1.19010561 & -0.00220115 & -0.01585757 & -0.00006562 \\
\hline
\end{tabular}
VII are available from the authors upon request.

TABLE VI. Coefficients for fits of the $g q$ scaling functions. 
TABLE VII. Coefficients for fits of the $g g$ scaling functions.

\begin{tabular}{|c|c|c|c|c|c|}
\hline \multirow[b]{2}{*}{$i$} & \multirow{2}{*}{$\begin{array}{r}f_{g g}^{(10)} \\
a_{i}\end{array}$} & \multicolumn{2}{|c|}{$f_{g g}^{(21)}$} & \multicolumn{2}{|c|}{$f_{g g}^{(22)}$} \\
\hline & & $b_{i}$ & $c_{i}$ & $b_{i}$ & $c_{i}$ \\
\hline 1 & -8.92563222 & -4.18931464 & 0.12306772 & 0.01222783 & -0.00380386 \\
\hline 2 & 149.90572830 & 82.35066406 & -2.75808806 & -0.77856184 & 0.08757766 \\
\hline 3 & -140.55601420 & -87.87311969 & 3.19739272 & 1.33955698 & -0.10742267 \\
\hline 4 & -0.34115615 & 9.80259328 & -0.56233045 & -0.59108409 & 0.02382706 \\
\hline 5 & -2.41049833 & -1.12268550 & 0.03240048 & 0.00248333 & -0.00099760 \\
\hline 6 & 54.73381889 & 29.51830225 & -0.92541788 & -0.23827213 & 0.02932941 \\
\hline 7 & 90.91548015 & 48.36110694 & -1.57154712 & -0.38868910 & 0.04906147 \\
\hline 8 & -4.88401008 & -7.06261770 & 0.35109760 & 0.28342153 & -0.01373734 \\
\hline 9 & -0.17466779 & -0.08025226 & 0.00227936 & 0.00010876 & -0.00006986 \\
\hline 10 & 13.47033628 & 7.01493779 & -0.21030153 & -0.03383862 & 0.00658371 \\
\hline 11 & 22.66482710 & 15.00588140 & -0.63688407 & -0.29071016 & 0.02089321 \\
\hline 12 & 4.60726682 & 3.84142441 & -0.12959776 & -0.11473654 & 0.00495414 \\
\hline 13 & -67.62342328 & -47.02161789 & 1.91690216 & 0.98929369 & -0.06553459 \\
\hline 14 & -9.70391427 & -8.05583379 & 0.26755747 & 0.24899069 & -0.01046635 \\
\hline 15 & 65.08050888 & 47.02740535 & -1.86154423 & -1.06096321 & 0.06559130 \\
\hline 16 & 5.09663260 & 4.21438052 & -0.13795865 & -0.13425338 & 0.00551218 \\
\hline 17 & -20.12225341 & -14.99599732 & 0.58155056 & 0.35935660 & -0.02095059 \\
\hline
\end{tabular}

$$
\begin{aligned}
h\left(\beta, a_{1}, \ldots, a_{17}\right)= & a_{1} \beta^{2}+a_{2} \beta^{3}+a_{3} \beta^{4}+a_{4} \beta^{5}+a_{5} \beta^{2} \ln \beta+a_{6} \beta^{3} \ln \beta+a_{7} \beta^{4} \ln \beta+a_{8} \beta^{5} \ln \beta+a_{9} \beta^{2} \ln ^{2} \beta \\
& +a_{10} \beta^{3} \ln ^{2} \beta+a_{11} \beta \ln \rho+a_{12} \beta \ln ^{2} \rho+a_{13} \beta^{2} \ln \rho+a_{14} \beta^{2} \ln ^{2} \rho+a_{15} \beta^{3} \ln \rho+a_{16} \beta^{3} \ln ^{2} \rho \\
& +a_{17} \beta^{4} \ln \rho,
\end{aligned}
$$

$h_{g q}^{(a)}\left(\beta, a_{1}, \ldots, a_{15}\right)=a_{1} \beta^{4}+a_{2} \beta^{5}+a_{3} \beta^{6}+a_{4} \beta^{4} \ln \beta+a_{5} \beta^{5} \ln \beta+a_{6} \beta^{6} \ln \beta+a_{7} \beta^{2} \rho \ln \rho+a_{8} \beta^{2} \rho \ln ^{2} \rho$$$
+a_{9} \beta^{3} \rho \ln \rho+a_{10} \beta^{3} \rho \ln ^{2} \rho+a_{11} \beta^{4} \rho \ln \rho+a_{12} \beta^{4} \rho \ln ^{2} \rho+a_{13} \beta^{2} \rho \ln ^{3} \rho+a_{14} \beta^{2} \rho \ln ^{4} \rho
$$$$
+a_{15} \beta^{2} \rho \ln ^{5} \rho \text {, }
$$

$$
\begin{aligned}
h_{g q}^{(b)}\left(\beta, a_{1}, \ldots, a_{18}\right)= & a_{1} \beta^{3}+a_{2} \beta^{4}+a_{3} \beta^{5}+a_{4} \beta^{6}+a_{5} \beta^{7}+a_{6} \beta^{4} \ln \beta+a_{7} \beta^{5} \ln \beta+a_{8} \beta^{6} \ln \beta+a_{9} \beta^{7} \ln \beta \\
& +a_{10} \beta^{3} \ln \rho+a_{11} \beta^{3} \ln ^{2} \rho+a_{12} \beta^{4} \ln \rho+a_{13} \beta^{4} \ln ^{2} \rho+a_{14} \beta^{5} \ln \rho+a_{15} \beta^{5} \ln ^{2} \rho+a_{16} \beta^{6} \ln \rho \\
& +a_{17} \beta^{6} \ln ^{2} \rho+a_{18} \beta^{7} \ln \rho
\end{aligned}
$$

[1] W. Bernreuther, J. Phys. G 35, 083001 (2008).

[2] J. R. Incandela et al., arXiv:0904.2499.

[3] S. Moch and P. Uwer, Phys. Rev. D 78, 034003 (2008).

[4] S. Moch and P. Uwer, Nucl. Phys. B, Proc. Suppl. 183, 75 (2008).

[5] N. Kidonakis and R. Vogt, Phys. Rev. D 78, 074005 (2008).

[6] M. Cacciari et al., J. High Energy Phys. 09 (2008) 127.

[7] P. M. Nadolsky et al., Phys. Rev. D 78, 013004 (2008).

[8] V. M. Abazov et al. (D0 Collaboration), arXiv:0903.5525.

[9] P. Nason, S. Dawson, and R. K. Ellis, Nucl. Phys. B303, 607 (1988).

[10] W. Beenakker et al., Phys. Rev. D 40, 54 (1989).

[11] W. Bernreuther et al., Nucl. Phys. B690, 81 (2004).
[12] M. Czakon and A. Mitov, arXiv:0811.4119.

[13] Tevatron Electroweak Working Group, arXiv:0903.2503.

[14] A. D. Martin et al., arXiv:0901.0002.

[15] N. Kidonakis et al., Phys. Rev. D 64, 114001 (2001).

[16] M. Cacciari et al., J. High Energy Phys. 04 (2004) 068.

[17] A. Petrelli et al., Nucl. Phys. B514, 245 (1998).

[18] K. Hagiwara, Y. Sumino, and H. Yokoya, Phys. Lett. B 666, 71 (2008).

[19] Y. Kiyo et al., Eur. Phys. J. C 60, 375 (2009).

[20] W. Beenakker et al., Nucl. Phys. B411, 343 (1994).

[21] W. Bernreuther, M. Fücker, and Z. G. Si, Phys. Rev. D 74, 113005 (2006).

[22] J. H. Kühn, A. Scharf, and P. Uwer, Eur. Phys. J. C 51, 37 (2007). 
[23] N. Gray et al., Z. Phys. C 48, 673 (1990).

[24] K. G. Chetyrkin and M. Steinhauser, Nucl. Phys. B573, 617 (2000).

[25] K. Melnikov and T. v. Ritbergen, Phys. Lett. B 482, 99 (2000).

[26] E. Laenen, L. Magnea, and G. Stavenga, Phys. Lett. B 669, 173 (2008).

[27] S. Moch and A. Vogt, J. High Energy Phys. 04 (2009) 081.

[28] S. Moch, J. A. M. Vermaseren, and A. Vogt, Nucl. Phys. B688, 101 (2004).

[29] A. Vogt, S. Moch, and J. A. M. Vermaseren, Nucl. Phys. B691, 129 (2004).

[30] M. Czakon, A. Mitov, and S. Moch, Nucl. Phys. B798, 210 (2008).

[31] M. Czakon, A. Mitov, and S. Moch, Phys. Lett. B 651, 147 (2007).

[32] J. G. Körner, Z. Merebashvili, and M. Rogal, Phys. Rev. D 77, 094011 (2008).

[33] B. Kniehl et al., Phys. Rev. D 78, 094013 (2008).

[34] C. Anastasiou and S. M. Aybat, Phys. Rev. D 78, 114006 (2008).
[35] R. Bonciani et al., arXiv:0810.0598.

[36] M. Czakon, Phys. Lett. B 664, 307 (2008).

[37] R. Bonciani et al., arXiv:0906.3671.

[38] R. Bonciani et al., Nucl. Phys. B529, 424 (1998).

[39] S. Dittmaier, P. Uwer, and S. Weinzierl, Phys. Rev. Lett. 98, 262002 (2007).

[40] S. Dittmaier, P. Uwer, and S. Weinzierl, Eur. Phys. J. C 59, 625 (2009).

[41] A. H. Hoang et al., Eur. Phys. J. direct C 2, 1 (2000).

[42] G. Rodrigo, A. Santamaria, and M. S. Bilenky, Phys. Rev. Lett. 79, 193 (1997).

[43] A. Brandenburg et al., Phys. Lett. B 468, 168 (1999).

[44] R. Barate et al. (ALEPH Collaboration), Eur. Phys. J. C 18, 1 (2000).

[45] G. Abbiendi et al. (OPAL Collaboration), Eur. Phys. J. C 21, 411 (2001).

[46] J. Abdallah et al. (DELPHI Collaboration), Eur. Phys. J. C 46, 569 (2006).

[47] J. Abdallah et al. (DELPHI Collaboration), Eur. Phys. J. C 55, 525 (2008).

[48] A. H. Hoang et al., Phys. Rev. Lett. 101, 151602 (2008). 